\title{
Starobinsky cosmological model in Palatini formalism
}

\author{
Aleksander Stachowski ${ }^{1, \mathrm{a}}$, Marek Szydłowski ${ }^{1,2, b}$, Andrzej Borowiec ${ }^{3, \mathrm{c}}$ \\ ${ }^{1}$ Astronomical Observatory, Jagiellonian University, Orla 171, 30-244 Kraków, Poland \\ ${ }^{2}$ Mark Kac Complex Systems Research Centre, Jagiellonian University, Łojasiewicza 11, 30-348 Kraków, Poland \\ ${ }^{3}$ Institute for Theoretical Physics, Wroclaw University, pl. Maxa Borna 9, 50-204 Wrocław, Poland
}

Received: 20 April 2017 / Accepted: 8 June 2017 / Published online: 17 June 2017

(C) The Author(s) 2017. This article is an open access publication

\begin{abstract}
We classify singularities in FRW cosmologies, which dynamics can be reduced to the dynamical system of the Newtonian type. This classification is performed in terms of the geometry of a potential function if it has poles. At the sewn singularity, which is of a finite scale factor type, the singularity in the past meets the singularity in the future. We show that such singularities appear in the Starobinsky model in $f(\hat{R})=\hat{R}+\gamma \hat{R}^{2}$ in the Palatini formalism, when dynamics is determined by the corresponding piecewise-smooth dynamical system. As an effect we obtain a degenerate singularity. Analytical calculations are given for the cosmological model with matter and the cosmological constant. The dynamics of model is also studied using dynamical system methods. From the phase portraits we find generic evolutionary scenarios of the evolution of the universe. For this model, the best fit value of $\Omega_{\gamma}=3 \gamma H_{0}^{2}$ is equal $9.70 \times 10^{-11}$. We consider a model in both Jordan and Einstein frames. We show that after transition to the Einstein frame we obtain both the form of the potential of the scalar field and the decaying Lambda term.
\end{abstract}

\section{Introduction}

The main aim of the paper is the construction of the Starobinsky model with a squared term $\hat{R}^{2}$ in the Palatini formalism and the investigation of cosmological implications of this model. In this model the inflation phase of evolution of the universe can be obtained by the modification of general relativity in the framework of $f(\hat{R})$ modified gravity theories [1]. In this context, historically the first theory of inflation was proposed by Starobinsky [2]. In the original Starobinsky model the term $R^{2} / 6 M^{2}$ was motivated by the conformal anomaly in the quantum gravity. The problem of

\footnotetext{
a e-mail: aleksander.stachowski@uj.edu.pl

b e-mail: marek.szydlowski@uj.edu.pl

${ }^{c}$ e-mail: andrzej.borowiec@ift.uni.wroc.pl
}

inflation in an $f(R)$ cosmological model is strictly related with the choice of frames. The authors of [1] show that CMB spectra in both Einstein and Jordan frames are different functions of the number of e-foldings until the end of inflation.

Inflation is a hypothesis about the existence of a short but very fast (of exponential type) accelerated growth of the scale factor $a(t)$ during the early evolution of the universe, after the Big-Bang but before the radiation-dominated epoch $[3,4]$. It implies $\ddot{a}(t)>0$. Irregularities in the early epoch may lead to the formation of structures in the universe due to the appearance of inflation.

Starobinsky [2] was the first who proposed a very simple theoretical model with one parameter $M$ (energy scale $M$ ) of such inflation and which is in good agreement with astronomical data and CMB observation. The Starobinsky model is representing the simplest version of $f(R)$ gravity theories which have been developed considerably in the last decade $[1,5,6]$, whose extra term in the Lagrangian is quadratic in the scalar curvature. This model predicts the value of spectral index $n_{s}=0.9603 \pm 0.0073$, at the $68 \% \mathrm{CL}$, with deviation from scale-invariance of the primordial power spectrum $[7,8]$.

The Starobinsky model is also compatible with Planck 2015 data [9] and nicely predicts the number $N=50-60$ e-folds between the start and the end of inflation [10].

It has been recently investigated some generalization of the Starobinsky inflationary model with a polynomial form of $f(R)=R+\frac{R^{2}}{6 M^{2}}+\frac{\lambda_{n}}{2 n} \frac{R^{n}}{\left(3 M^{2}\right)^{n-1}}$. It was demonstrated that the slow-roll inflation can be achieved as long as the dimensionless coupling $\lambda_{n}$ is sufficiently small [11].

The Starobinsky model becomes generic because the smallness of the dimensionless coupling constant $\lambda_{n}$ does not imply that fine-tuning is necessary [11]. The Starobinsky model was developed in many papers [8,12-17].

In this paper we develop the idea of endogenous inflation as an effect of modification of the FRW equation after 
the formulation of $f(R)$ cosmological model in the Palatini formalism.

We are looking for an inflation mechanism as a pure dynamical mechanism driven by the presence of the additional term (square of the Ricci scalar) in the Lagrangian, without necessity of the choice of a frame (Einstein vs. Jordan frame) [16-18].

In modern cosmology, a most popular trend is to explain the dark energy and the dark matter in terms of some substances, of which the nature is unknown up to now. Einstein was representing the opposite relational point of view on the description of gravity, in which all substantial forms should be eliminated. Such a point of view is called antisubstantialism. Extended $f(R)$ gravity models $[6,19]$ offer intrinsic or geometric models of both dark matter and dark energy - the key elements of Standard Cosmological Model. Therefore, the Einstein idea of relational gravity, in which dark matter and dark energy can be interpreted as geometric objects, is naturally realized in $f(\hat{R})$ extended gravity. The methods of dynamical system in the context of investigation dynamics of $f(R)$ models are used since Carroll $[19,20]$.

Unfortunately, the metric formulation of extended gravity gives rise to fourth order field equations. To avoid this difficulty, the Palatini formalism can be apply where both the metric $g$ and the symmetric connection $\Gamma$ are assumed to be independent dynamical variables. In consequence, one gets a system of second order partial differential equations. The Palatini approach reveals that the early universe inherits properties of the global $\Lambda \mathrm{CDM}$ evolution.

The Palatini approach has become of some interest lately. An excellent review of the Palatini $f(R)$ theories can be found in Olmo's paper [21]. He has published many other papers on this topic, namely, about the scalar-tensor representation of the Palatini theories [22,23]. The other important papers were on the existence of non-singular solutions in the Palatini gravity [24,25]. Some more recent papers concentrate on studying black holes and their singularities in the Palatini approach [26-30]. Other work which is important to mention is Flanagan's papers on the choice of a conformal frame [31,32]. Pannia et al. considered the impact of the Starobinsky model in compact stars [33].

In the Palatini gravity action for $f(\hat{R})$ gravity is introduced to be

$S=S_{\mathrm{g}}+S_{\mathrm{m}}=\frac{1}{2} \int \sqrt{-g} f(\hat{R}) \mathrm{d}^{4} x+S_{\mathrm{m}}$,

where $\hat{R}=g^{\mu \nu} \hat{R}_{\mu \nu}(\Gamma)$ is the generalized Ricci scalar and $\hat{R}_{\mu \nu}(\Gamma)$ is the Ricci tensor of a torsionless connection $\Gamma$. In this paper, we assume that $8 \pi G=c=1$. The equation of motion obtained from the first order Palatini formalism reduces to
$f^{\prime}(\hat{R}) \hat{R}_{\mu \nu}-\frac{1}{2} f(\hat{R}) g_{\mu \nu}=T_{\mu \nu}$

$\hat{\nabla}_{\alpha}\left(\sqrt{-g} f^{\prime}(\hat{R}) g^{\mu v}\right)=0$,

where $T_{\mu \nu}=-\frac{2}{\sqrt{-g}} \frac{\delta L_{\mathrm{m}}}{\delta g_{\mu \nu}}$ is matter energy-momentum tensor, i.e. one assumes that the matter minimally couples to the metric. As a consequence the energy-momentum tensor is conserved, i.e.: $\nabla^{\mu} T_{\mu \nu}=0$ [34]. In Eq. (3) $\hat{\nabla}_{\alpha}$ means the covariant derivative calculated with respect to $\Gamma$. In order to solve Eq. (3) it is convenient to introduce a new metric,

$\sqrt{h} h_{\mu \nu}=\sqrt{-g} f^{\prime}(\hat{R}) g_{\mu \nu}$

for which the connection $\Gamma=\Gamma_{\mathrm{LC}}(h)$ is a Levi-Civita connection. As a consequence in $\operatorname{dim} M=4$ one gets

$h_{\mu \nu}=f^{\prime}(\hat{R}) g_{\mu \nu}$,

i.e. both metrics are related by the conformal factor. For this reason one should assume that the conformal factor $f^{\prime}(\hat{R}) \neq$ 0 , so it has strictly positive or negative values.

Taking the trace of (2), we obtain additional so called structural equation

$f^{\prime}(\hat{R}) \hat{R}-2 f(\hat{R})=T$.

where $T=g^{\mu \nu} T_{\mu \nu}$. Because of cosmological applications we assume that the metric $g$ is FRW metric

$\mathrm{d} s^{2}=-\mathrm{d} t^{2}+a^{2}(t)\left[\frac{1}{1-k r^{2}} \mathrm{~d} r^{2}+r^{2}\left(\mathrm{~d} \theta^{2}+\sin ^{2} \theta \mathrm{d} \phi^{2}\right)\right]$,

where $a(t)$ is the scale factor, $k$ is a constant of spatial curvature $(k=0, \pm 1), t$ is the cosmological time. For simplicity of presentation we consider the flat model $(k=0)$.

As a source of gravity we assume a perfect fluid, with the energy-momentum tensor

$T_{v}^{\mu}=\operatorname{diag}(-\rho, p, p, p)$,

where $p=w \rho, w=$ const is a form of the equation of state ( $w=0$ for dust and $w=1 / 3$ for radiation). Formally, effects of the spatial curvature can also be included into the model by introducing a curvature fluid $\rho_{\mathrm{k}}=-\frac{k}{2} a^{-2}$, with the barotropic factor $w=-\frac{1}{3}\left(p_{\mathrm{k}}=-\frac{1}{3} \rho_{\mathrm{k}}\right)$. From the conservation condition $T_{v ; \mu}^{\mu}=0$ we obtain $\rho=\rho_{0} a^{-3(1+w)}$. Therefore the trace $T$ reads

$T=\sum_{i} \rho_{i, 0}\left(3 w_{i}-1\right) a(t)^{-3\left(1+w_{i}\right)}$. 
In what follows we consider visible and dark matter $\rho_{\mathrm{m}}$ in the form of dust $w=0$, dark energy $\rho_{\Lambda}$ with $w=-1$ and radiation $\rho_{\mathrm{r}}$ with $w=1 / 3$.

Because a form of the function $f(\hat{R})$ is unknown, one needs to probe it via ensuing cosmological models. Here we choose the simplest modification of the general relativity Lagrangian,

$f(\hat{R})=\hat{R}+\gamma \hat{R}^{2}$,

induced by the first three terms in the power series decomposition of an arbitrary function $f(R)$. In fact, since the terms $\hat{R}^{n}$ have different physical dimensions, i.e. $\left[\hat{R}^{n}\right] \neq\left[\hat{R}^{m}\right]$ for $n \neq m$, one should take instead the function $\hat{R}_{0} f\left(\hat{R} / \hat{R}_{0}\right)$ for constructing our Lagrangian, where $\hat{R}_{0}$ is a constant and $\left[\hat{R}_{0}\right]=[\hat{R}]$. In this case the power series expansion reads $\hat{R}_{0} f\left(\hat{R} / \hat{R}_{0}\right)=\hat{R}_{0} \sum_{n=0} \alpha_{n}\left(\hat{R} / \hat{R}_{0}\right)^{n}=\sum_{n=0} \tilde{\alpha}_{n} \hat{R}^{n}$, where the coefficients $\alpha_{n}$ are dimensionless, while $\left[\tilde{\alpha}_{n}\right]=[\hat{R}]^{1-n}$ are dimension full.

From the other hand the Lagrangian (10) can be viewed as a simplest deviation, by the quadratic Starobinsky term, from the Lagrangian $\hat{R}$ which provides the standard cosmological model a.k.a. $\Lambda$ CDM model. A corresponding solution of the structural equation (6)

$\hat{R}=-T \equiv 4 \rho_{\Lambda, 0}+\rho_{\mathrm{m}, 0} a^{-3}$.

is, in fact, exactly the same as for the $\Lambda \mathrm{CDM}$ model, i.e. with $\gamma=0$. However, the Friedmann equation of the $\Lambda \mathrm{CDM}$ model (with dust matter, dark energy and radiation)

$H^{2}=\frac{1}{3}\left(\rho_{\mathrm{r}, 0} a^{-4}+\rho_{\mathrm{m}, 0} a^{-3}+\rho_{\Lambda, 0}\right)$

is now hardly affected by the presence of quadratic term. More exactly a counterpart of the above formula in the model under consideration looks as follows:

$$
\begin{aligned}
\frac{H^{2}}{H_{0}^{2}}= & \frac{b^{2}}{\left(b+\frac{d}{2}\right)^{2}}\left[\Omega_{\gamma}\left(\Omega_{\mathrm{m}, 0} a^{-3}+\Omega_{\Lambda, 0}\right)^{2}\right. \\
& \times \frac{(K-3)(K+1)}{2 b}+\left(\Omega_{\mathrm{m}, 0} a^{-3}+\Omega_{\Lambda, 0}\right) \\
& \left.+\frac{\Omega_{\mathrm{r}, 0} a^{-4}}{b}+\Omega_{k}\right],
\end{aligned}
$$

where

$$
\begin{aligned}
& \Omega_{k}=-\frac{k}{H_{0}^{2} a^{2}}, \\
& \Omega_{\mathrm{r}, 0}=\frac{\rho_{\mathrm{r}, 0}}{3 H_{0}^{2}}, \\
& \Omega_{\mathrm{m}, 0}=\frac{\rho_{\mathrm{m}, 0}}{3 H_{0}^{2}},
\end{aligned}
$$

$\Omega_{\Lambda, 0}=\frac{\rho_{\Lambda, 0}}{3 H_{0}^{2}}$,

$K=\frac{3 \Omega_{\Lambda, 0}}{\left(\Omega_{\mathrm{m}, 0} a^{-3}+\Omega_{\Lambda, 0}\right)}$,

$\Omega_{\gamma}=3 \gamma H_{0}^{2}$,

$b=f^{\prime}(\hat{R})=1+2 \Omega_{\gamma}\left(\Omega_{\mathrm{m}, 0} a^{-3}+4 \Omega_{\Lambda, 0}\right)$,

$d=\frac{1}{H} \frac{\mathrm{d} b}{\mathrm{~d} t}=-2 \Omega_{\gamma}\left(\Omega_{\mathrm{m}, 0} a^{-3}+\Omega_{\Lambda, 0}\right)(3-K)$

From the above one can check that the standard model (12) can be reconstructed in the limit $\gamma \mapsto 0$. The study of this generalized Friedmann equation is a main subject of our research.

The paper is organized as follows. In Sect. 2, we consider the Palatini approach in the Jordan and Einstein frame. In Sect. 3, we present some generalities concerning dynamical systems of Newtonian type, and their relations with the Palatini-Starobinsky model. Section 4, is devoted to the classification of cosmological singularities with special attention on Newtonian type systems represented by potential function $V(a)$. We adopt the Fernandes-Jambrina and Lazkoz classification of singularities [35] to these systems using the notion of elasticity of the potential function with respect the scale factor. In Sect. 5, we will analyze the singularities in the Starobinsky model in the Palatini formalism. This system requires the form of piecewise-smooth dynamical system. Statistical analysis of the model is presented in Sect. 6. In Sect. 7, we shall summarize obtained results and draw some conclusions.

\section{The Palatini approach in different frames (Jordan vs. Einstein frame)}

Because the effect of acceleration can depend on a choice of a frame [36] this section is devoted to showing the existence of the inflation effect if the model is considered in the Einstein frame.

The action (1) is dynamically equivalent to the first order Palatini gravitational action, provided that $f^{\prime \prime}(\hat{R}) \neq 0[1,6$, 17]

$$
\begin{aligned}
S\left(g_{\mu \nu}, \Gamma_{\rho \sigma}^{\lambda}, \chi\right)= & \frac{1}{2} \int \mathrm{d}^{4} x \sqrt{-g}\left(f^{\prime}(\chi)(\hat{R}-\chi)+f(\chi)\right) \\
& +S_{m}\left(g_{\mu \nu}, \psi\right),
\end{aligned}
$$

Introducing a scalar field $\Phi=f^{\prime}(\chi)$ and taking into account the constraint $\chi=\hat{R}$, one gets the action (22) in the following form:

$$
\begin{aligned}
S\left(g_{\mu \nu}, \Gamma_{\rho \sigma}^{\lambda}, \Phi\right)= & \frac{1}{2} \int \mathrm{d}^{4} x \sqrt{-g}(\Phi \hat{R}-U(\Phi)) \\
& +S_{m}\left(g_{\mu \nu}, \psi\right),
\end{aligned}
$$


where the potential $U(\Phi)$ is defined by

$U_{f}(\Phi) \equiv U(\Phi)=\chi(\Phi) \Phi-f(\chi(\Phi))$

with $\Phi=\frac{\mathrm{d} f(\chi)}{\mathrm{d} \chi}$ and $\hat{R} \equiv \chi=\frac{\mathrm{d} U(\Phi)}{\mathrm{d} \Phi}$.

The Palatini variation of the action (23) gives rise to the following equations of motion:

$\Phi\left(\hat{R}_{\mu \nu}-\frac{1}{2} g_{\mu \nu} \hat{R}\right)+\frac{1}{2} g_{\mu \nu} U(\Phi)-T_{\mu \nu}=0$,

$\hat{\nabla}_{\lambda}\left(\sqrt{-g} \Phi g^{\mu \nu}\right)=0$,

$\hat{R}-U^{\prime}(\Phi)=0$.

Equation (25b) implies that the connection $\hat{\Gamma}$ is a metric connection for a new metric $\bar{g}_{\mu \nu}=\Phi g_{\mu \nu}$; thus $\hat{R}_{\mu \nu}=\bar{R}_{\mu \nu}, \bar{R}=$ $\bar{g}^{\mu \nu} \bar{R}_{\mu \nu}=\Phi^{-1} \hat{R}$ and $\bar{g}_{\mu \nu} \bar{R}=g_{\mu \nu} \hat{R}$. The $g$-trace of (25a) produces a new structural equation

$2 U(\Phi)-U^{\prime}(\Phi) \Phi=T$.

Now Eqs. (25a) and (25c) take the following form:

$\bar{R}_{\mu \nu}-\frac{1}{2} \bar{g}_{\mu \nu} \bar{R}=\bar{T}_{\mu \nu}-\frac{1}{2} \bar{g}_{\mu \nu} \bar{U}(\Phi)$,

$\Phi \bar{R}-\left(\Phi^{2} \bar{U}(\Phi)\right)^{\prime}=0$,

where we introduce $\bar{U}(\phi)=U(\phi) / \Phi^{2}, \bar{T}_{\mu \nu}=\Phi^{-1} T_{\mu \nu}$ and the structural equation can be replaced by

$\Phi \bar{U}^{\prime}(\Phi)+\bar{T}=0$

In this case, the action for the metric $\bar{g}_{\mu \nu}$ and scalar field $\Phi$ is given by

$S\left(\bar{g}_{\mu \nu}, \Phi\right)=\frac{1}{2} \int \mathrm{d}^{4} x \sqrt{-\bar{g}}(\bar{R}-\bar{U}(\Phi))+S_{m}\left(\Phi^{-1} \bar{g}_{\mu \nu}, \psi\right)$,

where we have to take into account a non-minimal coupling between $\Phi$ and $\bar{g}_{\mu \nu}$

$\bar{T}^{\mu \nu}=-\frac{2}{\sqrt{-\bar{g}}} \frac{\delta}{\delta \bar{g}_{\mu \nu}} S_{m}=(\bar{\rho}+\bar{p}) \bar{u}^{\mu} \bar{u}^{\nu}+\bar{p} \bar{g}^{\mu \nu}=\Phi^{-3} T^{\mu \nu}$,

$\bar{u}^{\mu}=\Phi^{-\frac{1}{2}} u^{\mu}, \bar{\rho}=\Phi^{-2} \rho, \bar{p}=\Phi^{-2} p, \bar{T}_{\mu \nu}=$ $\Phi^{-1} T_{\mu \nu}, \bar{T}=\Phi^{-2} T$ (see e.g. $[17,37]$ ).

In FRW case, one gets the metric $\bar{g}_{\mu \nu}$ in the following form:

$\mathrm{d} \bar{s}^{2}=-\mathrm{d} \bar{t}^{2}+\bar{a}^{2}(t)\left[\mathrm{d} r^{2}+r^{2}\left(\mathrm{~d} \theta^{2}+\sin ^{2} \theta \mathrm{d} \phi^{2}\right)\right]$ where $\mathrm{d} \bar{t}=\Phi(t)^{\frac{1}{2}} \mathrm{~d} t$ and new scale factor $\bar{a}(\bar{t})=$ $\Phi(\bar{t})^{\frac{1}{2}} a(\bar{t})$. Ensuing cosmological equations (in the case of the barotropic matter) are given by

$3 \bar{H}^{2}=\bar{\rho}_{\Phi}+\bar{\rho}_{m}, \quad 6 \frac{\ddot{\bar{a}}}{\bar{a}}=2 \bar{\rho}_{\Phi}-\bar{\rho}_{m}(1+3 w)$

where

$\bar{\rho}_{\Phi}=\frac{1}{2} \bar{U}(\Phi), \quad \bar{\rho}_{\mathrm{m}}=\rho_{0} \bar{a}^{-3(1+w)} \Phi^{\frac{1}{2}(3 w-1)}$

and $w=\bar{p}_{\mathrm{m}} / \bar{\rho}_{\mathrm{m}}=p_{\mathrm{m}} / \rho_{\mathrm{m}}$. In this case, the conservation equations has the following form:

$\dot{\bar{\rho}}_{\mathrm{m}}+3 \bar{H} \bar{\rho}_{\mathrm{m}}(1+w)=-\dot{\bar{\rho}}_{\Phi}$

Let us consider the Starobinsky-Palatini model in the above framework. The potential $\bar{U}$ is described by the following formula:

$\bar{U}(\Phi)=2 \bar{\rho}_{\Phi}(\Phi)=\left(\frac{1}{4 \gamma}+2 \lambda\right) \frac{1}{\Phi^{2}}-\frac{1}{2 \gamma} \frac{1}{\Phi}+\frac{1}{4 \gamma}$.

Figure 1 presents the relation $\bar{\rho}_{\Phi}(\Phi)$. Note that the function $\bar{\rho}_{\Phi}$ has the same shape like the Starobinsky potential. The function $\bar{\rho}_{\Phi}(\Phi)$ has the minimum for

$\Phi_{\min }=1+8 \gamma \lambda$

In general, the scalar field $\Phi(\bar{a})$ is given by (cf. (11))

$\Phi=1+2 \gamma \hat{R}=1+8 \gamma \lambda+2 \gamma \rho_{m}-6 \gamma p_{m}$.

Because $\bar{\rho}_{m}=\Phi^{-2} \rho_{m}, \quad \bar{p}_{m}=\Phi^{-2} p_{m}$, and taking into account (34) one gets

$2 \gamma(1-3 w) \rho_{0} \bar{a}^{-3(1+w)} \Phi^{\frac{3}{2}(w+1)}-\Phi+1+8 \gamma \lambda=0$.

the algebraic equation determining the function $\Phi(\bar{a})$ for a given barotropic factor $w$. This provides an implicit dependence $\Phi(\bar{a})$. In order to get it more explicit one needs to solve (39) for some interesting values $w$. For example in the case of dust we obtain the third order polynomial equation

$\left(\frac{1}{2 \gamma}+4 \Lambda\right) y^{3}-\frac{1}{2 \gamma} y+\rho_{0 w} \bar{a}^{-3}=0$

where $y=\Phi^{-\frac{1}{2}}$.

The evolution of $\Phi(\bar{a})$, at the beginning of the inflation epoch, is presented in Fig. 2.

For $\gamma \approx 0$, the potential $\bar{U}$ can be approximated as $\bar{U}=$ $-\bar{\rho}_{m}+\frac{1}{4 \gamma}$. In this case the Friedmann equation can be written as

$3 \bar{H}^{2}=\frac{\bar{\rho}_{m}}{2}+\frac{1}{8 \gamma}$. 


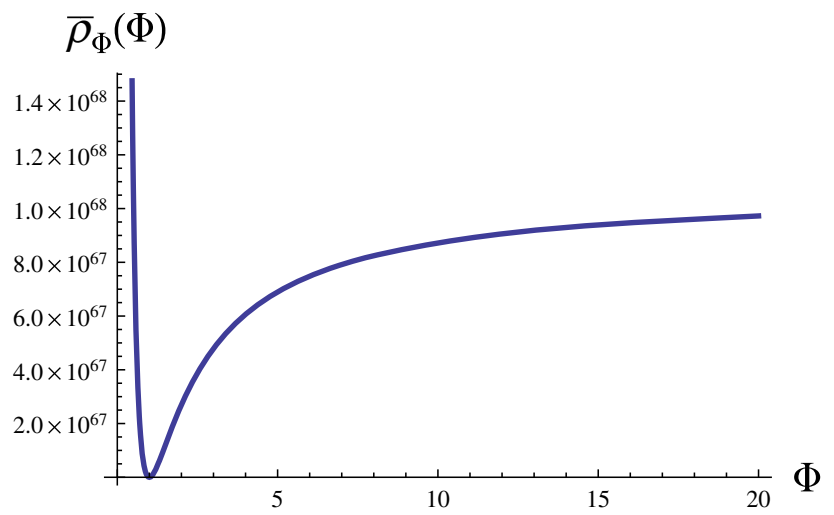

Fig. 1 Illustration of the dependence $\bar{\rho}_{\Phi}$ of $\Phi$. We assume that $\gamma=$ $1.16 \times 10^{-69} \mathrm{~s}^{2} \cdot \bar{\rho}_{\Phi}$ is expressed in units of $\frac{\mathrm{km}^{2}}{\mathrm{~s}^{2} \mathrm{Mpc}^{2}}$. Note that this potential has the same shape like the Starobinsky potential

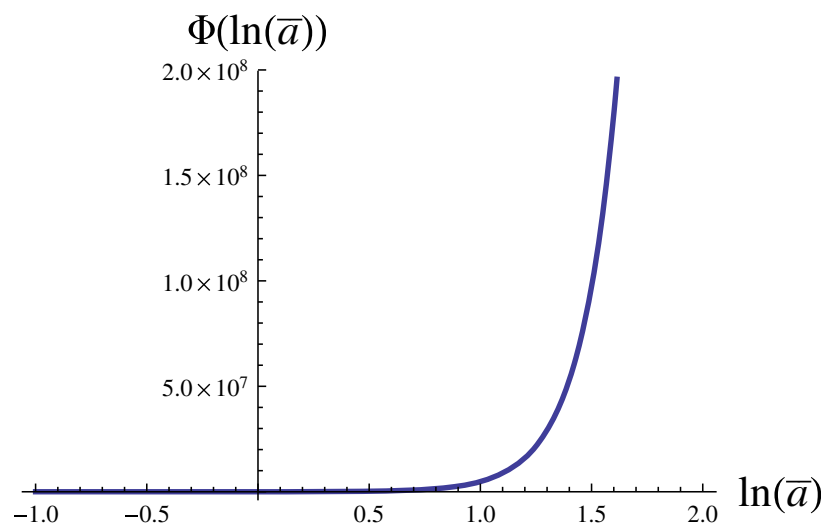

Fig. 2 Illustration of the typical evolution of $\Phi$ with respect to $\ln (\bar{a})$ at the beginning of the inflation epoch. We assume that $\gamma=1.16 \times$ $10^{-69} \mathrm{~s}^{2}$ and $\bar{a}_{0}=1$ at the beginning of the inflation epoch

In the case of $\bar{\rho}_{m}=0, \bar{\rho}_{\Phi}$ is constant and the Friedmann equation has the following form:

$3 \bar{H}^{2}=\frac{1}{8 \gamma}$.

In this model the inflation phenomenon appears when the value of the parameter $\gamma$ is close to zero and the matter $\bar{\rho}_{m}$ is negligible with comparison to $\bar{\rho}_{\Phi}$. In this case the approximate number of e-foldings is given by the following formula:

$N=H_{\text {init }}\left(\bar{t}_{\text {fin }}-\bar{t}_{\text {init }}\right)=\frac{\bar{t}_{\text {fin }}-\bar{t}_{\text {init }}}{\sqrt{24 \gamma}}$.

The number of e-folds $N$ should be equal $50 \sim 60$ in the inflation epoch [10]. In this model we obtain $N=60$, when $\gamma=1.16 \times 10^{-69} \mathrm{~s}^{2}$ and the timescale of the inflation is equal $10^{-32} \mathrm{~s}$ [38]. The relation between $\gamma$ and the approximate number of e-foldings $N$ is presented in Fig. 3 .

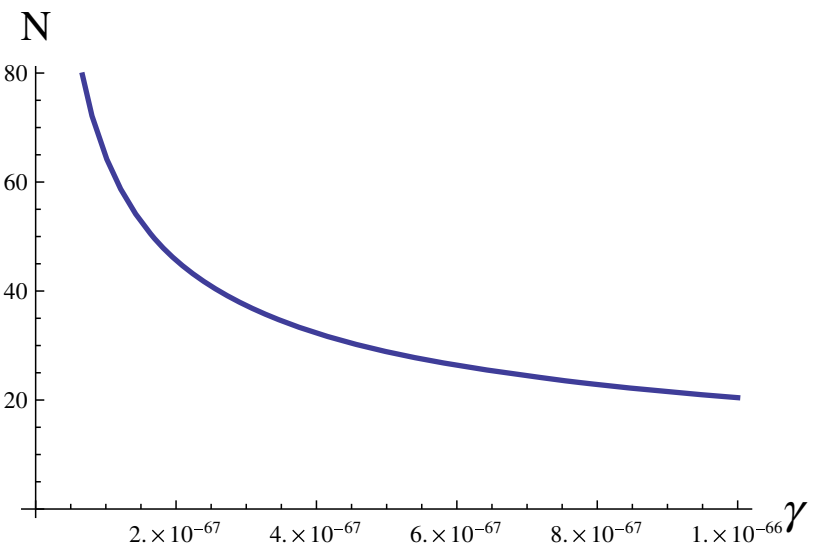

Fig. 3 The diagram of the relation between $\gamma$ and the approximate number of e-foldings $N=\bar{H}_{\text {init }}\left(\bar{t}_{\text {fin }}-\bar{t}_{\text {init }}\right)$ from $\bar{t}_{\text {init }}$ to $\bar{t}_{\text {fin }}$. We assume that $\bar{t}_{\text {fin }}-\bar{t}_{\text {init }} \approx 10^{-32} \mathrm{~s}$. The parameter $\gamma$ is expressed in units of $\mathrm{s}^{2}$. Note that the number of e-foldings grows when the parameter $\gamma$ decreases and $N=60$ when $\gamma=1.16 \times 10^{-69} \mathrm{~s}^{2}$

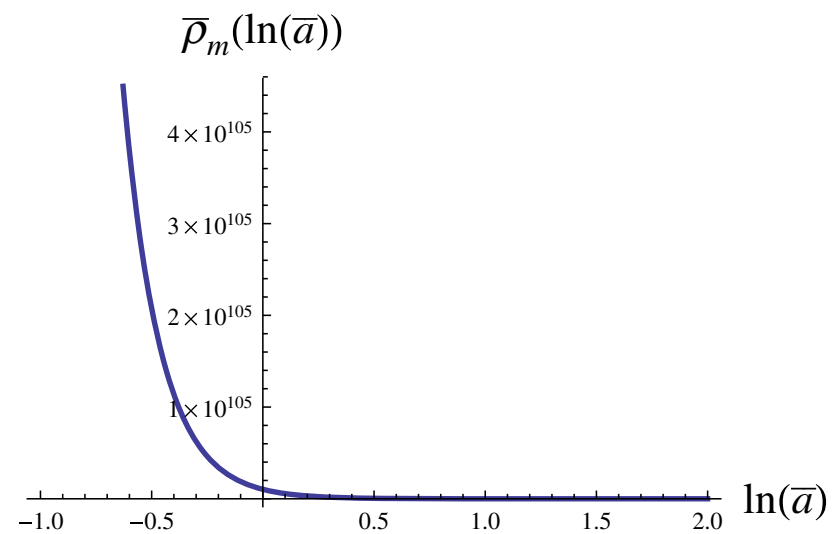

Fig. 4 Illustration of the typical evolution of $\bar{\rho}_{m}$ with respect to $\ln (\bar{a})$ at the beginning of the inflation epoch. We assume that $\gamma=1.16 \times$ $10^{-69} \mathrm{~s}^{2}$ and $\bar{a}_{0}=1$ at the beginning of the inflation epoch. $\bar{\rho}_{m}$ is expressed in units of $\frac{\mathrm{km}^{2}}{\mathrm{~s}^{2} \mathrm{Mpc}^{2}}$

The condition for appearing of the inflation is for the value of the parameter $\gamma$ to be close to zero, hence the influence of the parameter $\lambda$ on the evolution of the universe is negligible.

In Fig. 4 the typical evolution is demonstrated of $\bar{\rho}_{m}(\bar{a})$ at the beginning of the inflation epoch. The typical evolution of $\bar{\rho}_{\Phi}$, at the beginning of the inflation epoch, is presented in Fig. 5. Note that, for the late time universe, $\bar{\rho}_{\Phi}$ can be approximated as a constant. Figure 6 presents the evolution of the scale factor $\bar{a}(\bar{t})$ during the inflation. Figure 7 shows the Hubble function $\bar{H}$ during the inflation epoch.

The conservation equation for $\bar{\rho}_{\Phi}$ can be written

$\dot{\bar{\rho}}_{\Phi}=-3 \bar{H}\left(\bar{\rho}_{\Phi}+\bar{p}_{\Phi}\right)$

where $\bar{p}_{\Phi}$ is an effective pressure. In this case the equation of state for the dark energy is expressed by the following formula: 


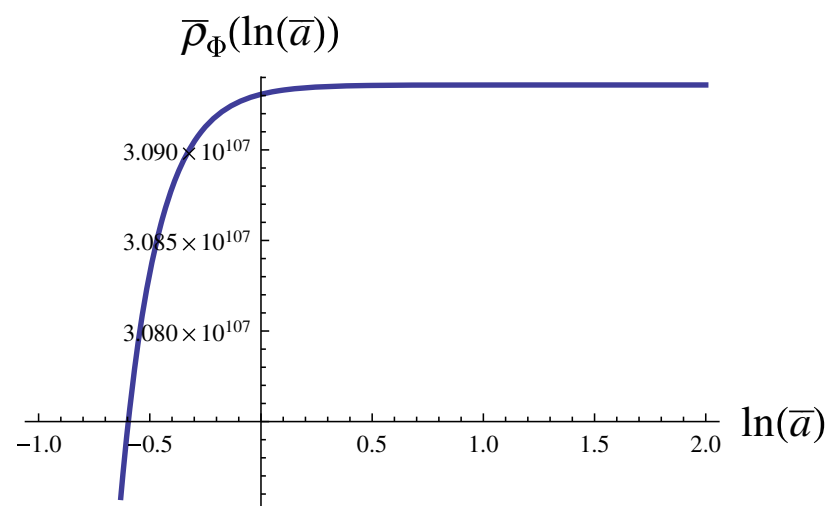

Fig. 5 Illustration of the typical evolution of $\bar{\rho}_{\phi}$ with respect to $\ln (\bar{a})$ at the beginning of the inflation epoch. We assume that $\gamma=1.16 \times$ $10^{-69} \mathrm{~s}^{2}$ and $\bar{a}_{0}=1$ at the beginning of the inflation epoch. $\bar{\rho}_{\Phi}$ is expressed in units of $\frac{\mathrm{km}^{2}}{\mathrm{~s}^{2} \mathrm{Mpc}^{2}}$. Note that during the inflation $\bar{\rho}_{\phi} \approx$ const

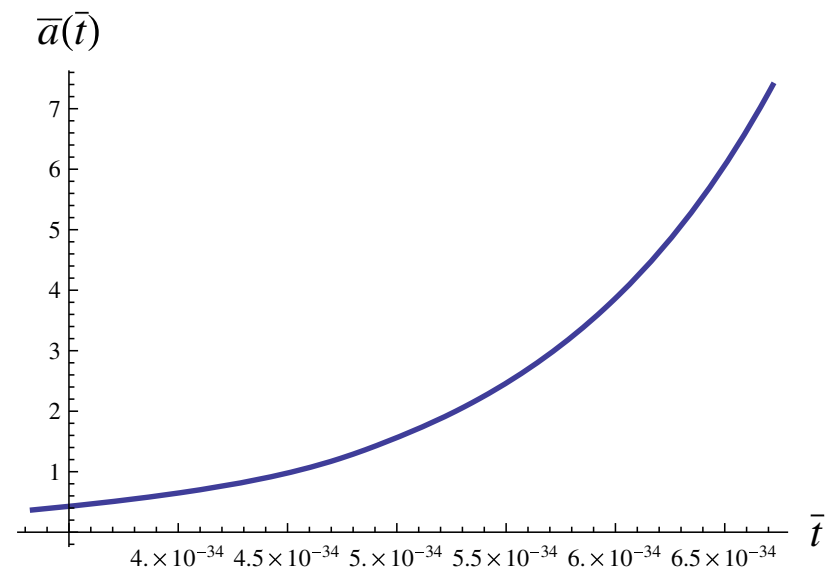

Fig. 6 Illustration of the typical evolution of $\bar{a}$ with respect to $\bar{t}$ at the beginning of the inflation epoch. We assume that $\gamma=1.16 \times$ $10^{-69} \mathrm{~s}^{2}$ and $\bar{a}_{0}=1$ at the beginning of the inflation epoch. The time $\bar{t}$ is expressed in seconds

$\bar{p}_{\Phi}=w(a) \bar{\rho}_{\Phi}$

where the function $w(a)$ is given by the expression

$w(a)=-1-\frac{\dot{\bar{\rho}}_{\Phi}}{\sqrt{3} \sqrt{\bar{\rho}_{\mathrm{m}}+\bar{\rho}_{\Phi}} \rho_{\Phi}}=-1-\frac{1}{3 \bar{H}} \frac{\mathrm{d} \ln \rho_{\Phi}}{\mathrm{d} \bar{t}}$.

The diagram of the coefficient of equation of state $w(a)$, at the beginning the inflation epoch, is presented in Fig. 8. Note that the function $w(a)$, for the late time, is a constant and equal -1 .

The action (23) can be rewritten in the Jordan frame $\left(g_{\mu \nu}, \Phi\right)$ as

$S=\frac{1}{2 k} \int \mathrm{d}^{4} x \sqrt{-g}\left(\Phi R+\frac{3}{2 \Phi} \partial_{\mu} \Phi \partial^{\mu} \Phi-U(\Phi)\right)$,

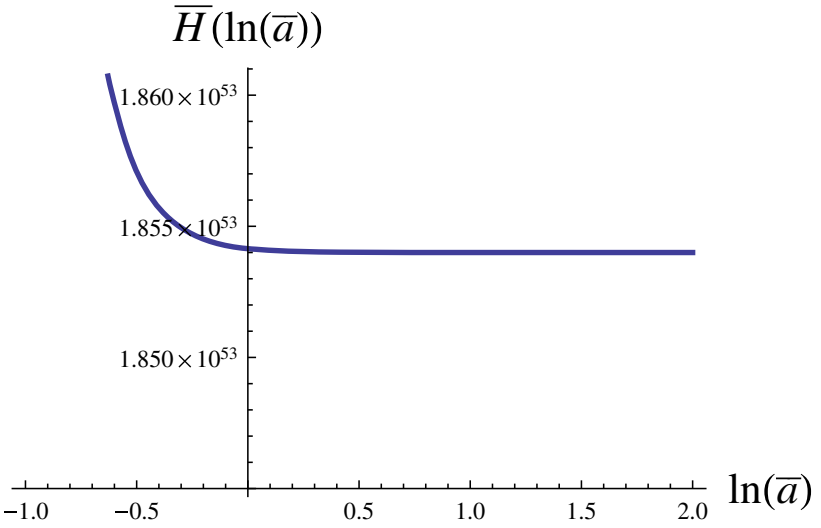

Fig. 7 Illustration of the typical evolution of $\bar{H}$ with respect to $\ln (\bar{a})$ at the beginning of the inflation epoch. We assume that $\gamma=1.16 \times$ $10^{-69} \mathrm{~s}^{2}$ and $\bar{a}_{0}=1$ at the beginning of the inflation epoch. $\bar{H}$ is expressed in units of $\frac{\mathrm{km}}{\mathrm{s} \mathrm{Mpc}}$. Note that, for the late time, $\bar{H}$ can be treated as a constant

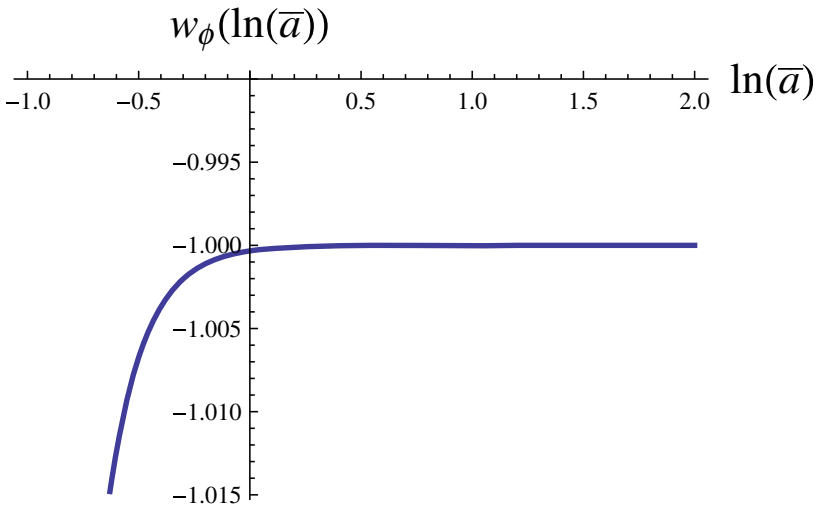

Fig. 8 Illustration of the typical evolution of $w_{\phi}$ with respect to $\ln (\bar{a})$. We assume that $\gamma=1.16 \times 10^{-69} \mathrm{~s}^{2}$ and $\bar{a}_{0}=1$ at the beginning of the inflation epoch. Note that during the inflation $w_{\phi} \approx-1$

where $R$ is the metric Ricci scalar, $\Phi=f^{\prime}(\hat{R}), \hat{R}=\chi(\Phi)$.

We obtain the Brans-Dicke action with the coupling parameter $\omega=-\frac{3}{2}$ in the Jordan frame. The equations of motion take the form

$$
\begin{aligned}
& \Phi\left(R_{\mu \nu}-\frac{1}{2} g_{\mu \nu} R\right)-\frac{3}{4 \Phi} g_{\mu \nu} \nabla_{\sigma} \Phi \nabla^{\sigma} \Phi+\frac{3}{2 \Phi} \nabla_{\mu} \Phi \nabla_{\nu} \Phi \\
& \quad+g_{\mu \nu} \square \Phi-\nabla_{\mu} \nabla_{\nu} \Phi+\frac{1}{2} g_{\mu \nu} U(\phi)-\kappa T_{\mu \nu}=0, \quad(47 \mathrm{a})
\end{aligned}
$$

$R-\frac{3}{\Phi} \square \Phi+\frac{3}{2 \Phi^{2}} \nabla_{\mu} \Phi \nabla^{\mu} \Phi-\frac{1}{2} U^{\prime}(\Phi)=0$.

In this case the dynamics of the metric $g$ is exactly the same as described by the original Palatini equations (2)-(6). On cosmological grounds it means that the scale factor $a(t)$ evolves according to the Friedmann equation (13). It has recently been shown that cosmological data favor the value $\omega \approx-1$ on the $3 \sigma$ level [39]. 


\section{Singularities in cosmological dynamical systems of Newtonian type}

There is a class of cosmological models, of which the dynamics can be reduced to a dynamical system of the Newtonian type. Let consider a homogeneous and isotropic universe with a spatially flat space-time metric of the form

$\mathrm{d} s^{2}=\mathrm{d} t^{2}-a^{2}(t)\left[\mathrm{d} r^{2}+r^{2}\left(\mathrm{~d} \theta^{2}+\sin ^{2} \theta \mathrm{d} \phi^{2}\right)\right]$,

where $a(t)$ is the scale factor and $t$ is the cosmological time.

Let us consider the energy-momentum tensor $T_{\nu}^{\mu}$ for the perfect fluid with energy density $\rho(t)$ and pressure $p(t)$ as a source of gravity. In this case the Einstein equations assume the form of the Friedmann equations,

$\rho=3 H^{2}=\frac{3 \dot{a}^{2}}{a^{2}}$,

$p=-\frac{2 \ddot{a}}{a}-\frac{\dot{a}^{2}}{a^{2}}$,

where dot denotes differentiation with respect to the cosmic time $t, H \equiv \frac{\dot{a}}{a}$ is the Hubble function. In our notation we use the natural system of units in which $8 \pi G=c=1$.

We assume $\rho(t)=\rho(a(t))$ as well as $p(t)=p(a(t))$, i.e. both energy density and pressure depend on the cosmic time through the scale factor $a(t)$. The conservation condition $T_{; \mu}^{\mu \nu}=0$ reduces to

$\dot{\rho}=-3 H(\rho+p)$.

It would be convenient to rewrite (49) in the equivalent form

$\dot{a}^{2}=-2 V(a)$,

where

$V(a)=-\frac{\rho(a) a^{2}}{6}$.

In (53) $\rho(a)$ plays the model role of an effective energy density. For example for the standard cosmological model (12)

$V=-\frac{\rho_{\mathrm{eff}} a^{2}}{6}=-\frac{a^{2}}{6}\left(\rho_{m, 0} a^{-3}+\rho_{\Lambda, 0}\right)$,

where $\rho_{\text {eff }}=\rho_{\mathrm{m}}+\Lambda$ and $\rho_{\mathrm{m}}=\rho_{\mathrm{m}, 0} a^{-3}$. Equation (50) is equivalent to

$\frac{\ddot{a}}{a}=-\frac{1}{6}(\rho+3 p)$, which is called the acceleration equation. It is easily to check that

$\ddot{a}=-\frac{\partial V}{\partial a}$,

where $V(a)$ is given by (53) provided that the conservation equation (51) is fulfilled.

Due to Eq. (56) the evolution of the universe can be interpreted as the motion of a fictitious particle of unit mass in the potential $V(a)$. Here $a(t)$ plays the role of a position variable. The equation of motion (56) assumes a form analogous to the Newtonian equation of motion.

If we know the form of the effective energy density then we can construct the form of the potential $V(a)$, which determines the whole dynamics in the phase space $(a, \dot{a})$. In this space the Friedmann equation (52) plays the role of a first integral and determines the phase space curves representing the evolutionary paths of the cosmological models. The diagram of the potential $V(a)$ contains all information needed to construction a phase space portrait. In this case the phase space is two-dimensional,

$\left\{(a, \dot{a}): \frac{\dot{a}^{2}}{2}+V(a)=-\frac{k}{2}\right\}$.

In the general case of an arbitrary potential, the dynamical system which describes the evolution of a universe takes the form

$\dot{a}=x$,

$\dot{x}=-\frac{\partial V(a)}{\partial a}$.

We shall study the system above using the theory of piecewise-smooth dynamical systems. Therefore it is assumed that the potential function, except some isolated (singular) points, belongs to the class $C^{2}\left(\mathbb{R}_{+}\right)$.

The lines $\frac{x^{2}}{2}+V(a)=-\frac{k}{2}$ represent possible evolutions of the universe for different initial conditions. Equations (58) and (59) can be rewritten in terms of dimensionless variables if we replace the effective energy density $\rho_{\text {eff }}$ by the density parameter:

$\Omega_{\mathrm{eff}}=\frac{\rho_{\mathrm{eff}}}{3 H_{0}^{2}}$.

Then

$\frac{1}{H_{0}^{2}} \frac{\dot{a}^{2}}{2}=-\frac{\Omega_{\mathrm{eff}} a^{2}}{2}$,

$\frac{\mathrm{d}^{2} a}{\mathrm{~d} \tau^{2}}=-\frac{\partial \tilde{V}}{\partial a}$, 
where $t \rightarrow \tau=\left|H_{0}\right| t$ and

$\tilde{V}(a)=-\frac{\Omega_{\mathrm{eff}} a^{2}}{2}$.

Any cosmological model can be identified by its form of the potential function $V(a)$ depending on the scale factor $a$. From the Newtonian form of the dynamical system (58)-(59) one can see that all critical points correspond to vanishing of r.h.s. of the dynamical system $\left(x_{0}=0,\left.\frac{\partial V(a)}{\partial a}\right|_{a=a_{0}}\right)$. Therefore all critical points are localized on the $x$-axis, i.e. they represent a static universe.

Because of the Newtonian form of the dynamical system the character of critical points is determined from the characteristic equation of the form

$a^{2}+\left.\operatorname{det} A\right|_{x_{0}=0,\left.\frac{\partial V(a)}{\partial a}\right|_{a_{0}}=0}=0$

where $\operatorname{det} A$ is the determinant of the linearization matrix calculated at the critical points, i.e.

$\operatorname{det} A=\left.\frac{\partial^{2} V(a)}{\partial a^{2}}\right|_{a_{0},\left.\frac{\partial V(a)}{\partial a}\right|_{a_{0}}=0}$.

From Eqs. (64) and (65) one can conclude that only admissible critical points are of saddle type if $\left.\frac{\partial^{2} V(a)}{\partial a^{2}}\right|_{a=a_{0}}<0$ or of center type if $\left.\frac{\partial^{2} V(a)}{\partial a^{2}}\right|_{a=a_{0}}>0$.

If the shape of the potential function is known (from knowledge of the effective energy density), then it is possible to calculate the cosmological functions in exact form,

$t=\int^{a} \frac{\mathrm{d} a}{\sqrt{-2 V(a)}}$

$H(a)= \pm \sqrt{-\frac{2 V(a)}{a^{2}}}$,

the deceleration parameter, the effective barotropic factor

$q=-\frac{a \ddot{a}}{\dot{a}^{2}}=\frac{1 \mathrm{~d} \ln (-V)}{2}$,

$w_{\text {eff }}(a(t))=\frac{p_{\text {eff }}}{\rho_{\text {eff }}}=-\frac{1}{3}\left(\frac{\mathrm{d} \ln (-V)}{\mathrm{d} \ln a}+1\right)$,

the parameter of deviation from de Sitter universe [35]

$h(t) \equiv-(q(t)+1)=\frac{1}{2} \frac{\mathrm{d} \ln (-V)}{\mathrm{d} \ln a}-1$

(note that if $V(a)=-\frac{\Lambda a^{2}}{6}, h(t)=0$ ), the effective matter density and pressure

$\rho_{\mathrm{eff}}=-\frac{6 V(a)}{a^{2}}$ $p_{\text {eff }}=\frac{2 V(a)}{a^{2}}\left(\frac{\mathrm{d} \ln (-V)}{\mathrm{d} \ln a}+1\right)$,

and, finally, the Ricci scalar curvature for the FRW metric (48),

$R=\frac{6 V(a)}{a^{2}}\left(\frac{\mathrm{d} \ln (-V)}{\mathrm{d} \ln a}+2\right)$

From the formulas above one can observe that the most of them depend on the quantity

$I_{v}(a)=\frac{\mathrm{d} \ln (-V)}{\mathrm{d} \ln a}$

This quantity measures the elasticity of the potential function, i.e. indicates how the potential $V(a)$ changes if the scale factor $a$ changes. For example, for the de Sitter universe $-V(a) \propto a^{2}$, the rate of growth of the potential is $2 \%$ as the rate of growth of the scale factor is $1 \%$.

In the classification of the cosmological singularities by Fernandez-Jambrina and Lazkoz [35] a crucial role is played by the parameter $h(t)$. Note that in a cosmological sense this parameter is

$h(t)=\frac{1}{2} I_{v}(a)-1$.

In this approach the classification of singularities is based on generalized power and asymptotic expansion of the barotropic index $w$ in the equation of state (or equivalently of the deceleration parameter $q$ ) in terms of the time coordinate.

\section{Degenerated singularities-new type (VI) of singularity—sewn singularities}

Recently, due to the discovery of an accelerated phase in the expansion of our universe, many theoretical possibilities for future singularities are seriously considered. If we assume that the universe expands following the Friedmann equation, then this phase of expansion is driven by dark energy-a hypothetical fluid, which violates the strong energy condition. Many of the new types of singularities were classified by Nojiri et al. [40]. Following their classification the type of singularity depends on the singular behavior of the cosmological quantities like the scale factor $a$, the Hubble parameter $H$, the pressure $p$ and the energy density $\rho$ :

- Type 0: 'Big crunch'. In this type, the scale factor $a$ is vanishing and there is blow-up of the Hubble parameter $H$, energy density $\rho$ and pressure $p$. 
- Type I: 'Big rip'. In this type, the scale factor $a$, energy density $\rho$ and pressure $p$ are blown up.

- Type II: 'Sudden'. The scale factor $a$, energy density $\rho$ and Hubble parameter $H$ are finite and $\dot{H}$ and the pressure $p$ are divergent.

- Type III: 'Big freeze'. The scale factor $a$ is finite and the Hubble parameter $H$, energy density $\rho$ and pressure $p$ are blown up [41] or divergent [42].

- Type IV. The scale factor $a$, Hubble parameter $H$, energy density $\rho$, pressure $p$ and $\dot{H}$ are finite but higher derivatives of the scale factor $a$ diverge.

- Type V. The scale factor $a$ is finite but the energy density $\rho$ and pressure $p$ vanish.

Following Królak [43], big rip and big crunch singularities are strong whereas sudden, big freeze and type IV are weak singularities.

In the model under consideration the potential function and/or its derivative can diverge at isolated points (value of the scale factor). Therefore the classification mentioned before has application only for a single component of piecewise-smooth potential. In our model the dynamical system describing the evolution of a universe belongs to the class of a piecewise-smooth dynamical systems. As a consequence new types of singularities at finite scale factor $a_{s}$ can appear for which $\frac{\partial V}{\partial a}\left(a_{s}\right)$ does not exist (is not determined). This implies that the classification of singularities should be extended to the case of non-isolated singularities.

Let us illustrate this idea on the example of a freeze singularity in the Starobinsky model with the Palatini formalism (previous section). Such a singularity has a complex character and in analogy to the critical point we called it degenerate. Formally it is composed of two types III singularities: one in the future and another one in the past. If we consider the evolution of the universe before this singularity we detect an isolated singularity of type III in the future. Conversely if we consider the evolution after the singularity, then going back in time we meet a type III singularity in the past. Finally, at the finite scale factor the two singularities will meet. For a description of behavior near the singularity one considers the $t=t(a)$ relation. This relation has a horizontal inflection point and it is natural to expand this relation in a Taylor series near this point at which $\frac{\mathrm{d} t}{\mathrm{~d} a}=\frac{1}{H a}$ is zero. For the freeze singularity, the scale factor remains constant $a_{\mathrm{s}}, \rho$ and $H$ blow up and $\ddot{a}$ is undefined. It this case, the degenerate singularity of type III is called sewn (non-isolated) singularity. We, therefore, obtain [44]

$t-t_{\mathrm{s}} \simeq \pm\left.\frac{1}{2} \frac{\mathrm{d}^{2} t}{\mathrm{~d} a^{2}}\right|_{a=a_{\text {sing }}}\left(a-a_{\text {sing }}\right)^{2}$.

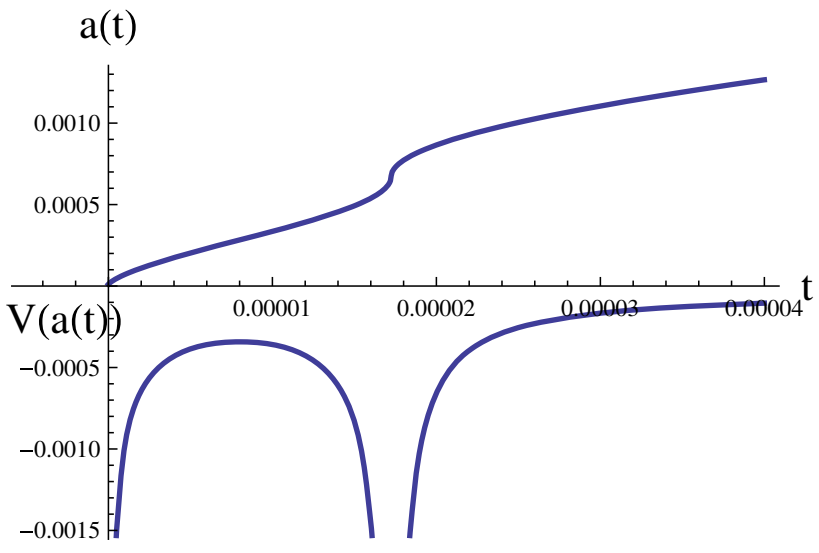

Fig. 9 Illustration of sewn freeze singularity, when the potential $V(a)$ has a pole

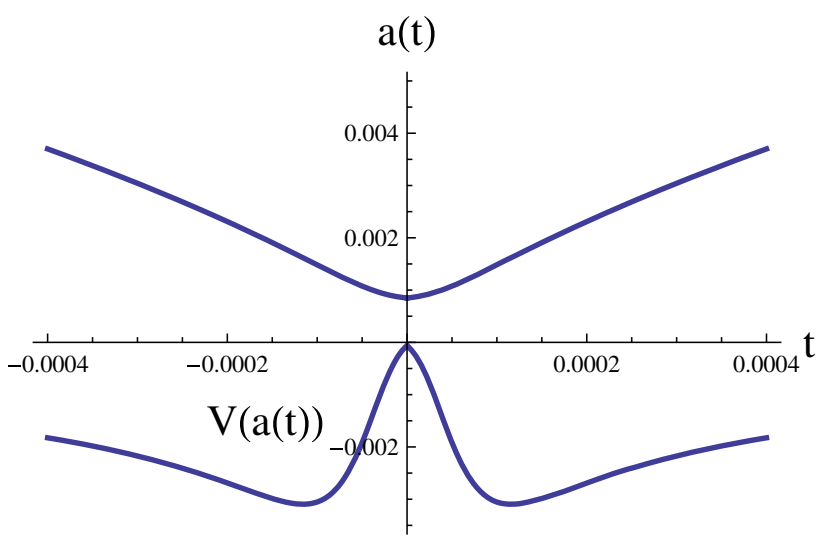

Fig. 10 Illustration of a sewn sudden singularity. The model with negative $\Omega_{\gamma}$ has a mirror symmetry with respect to the cosmological time. Note that the spike on the diagram shows a discontinuity of the function $\frac{\partial V}{\partial a}$. Note the existence of a bounce at $t=0$

The above formula combines two types of behavior near the freeze singularities in the future,

$a-a_{\text {sing }} \propto-\left(t_{\text {sing }}-t\right)^{1 / 2}$ for $t \rightarrow t_{\text {sing }^{-}}$

and in the past

$a-a_{\text {sing }} \propto+\left(t-t_{\text {sing }}\right)^{1 / 2}$ for $t \rightarrow t_{\text {sing }^{+}}$.

Figure 9 illustrates the behavior of the scale factor in cosmological time in neighborhood of a pole of the potential function. Diagram of $a(t)$ is constructed from the dynamics in two disjoint region $\left\{a: a<a_{\mathrm{s}}\right\}$ and $\left\{a: a>a_{\mathrm{s}}\right\}$. Figure 10 presents the behavior of the scale factor in the cosmological time in a neighborhood of the sudden singularity.

In the model under consideration another type of sewn singularity also appears. It is a composite singularity with two sudden singularities glued at the bounce when $a=a_{\min }$. In this singularity the potential itself is a continuous func- 
tion while its first derivative has a discontinuity. Therefore, the corresponding dynamical system represents a piecewisesmooth dynamical system.

The problem of $C^{0}$ metric extension beyond the future Cauchy horizon, when the second derivative of the metric is inextendible, was discussed in work of Sbierski [45]. In the context of FLRW cosmological models, Sbierski's methodology was considered in [46].

\section{Singularities in the Starobinsky model in the Palatini formalism}

In our model, one finds two types of singularities, which are a consequence of the Palatini formalism: the freeze and sudden singularity. The freeze singularity appears when the multiplicative expression $\frac{b}{b+d / 2}$, in the Friedmann equation (13), is equal to infinity. So we get a condition for the freeze singularity: $2 b+d=0$, which produces a pole in the potential function. It appears that the sudden singularity appears in our model when the multiplicative expression $\frac{b}{b+d / 2}$ vanishes. This condition is equivalent to the case $b=0$.

The freeze singularity in our model is a solution of the algebraic equation

$2 b+d=0 \Longrightarrow f\left(K, \Omega_{\Lambda, 0}, \Omega_{\gamma}\right)=0$

or

$-3 K-\frac{K}{3 \Omega_{\gamma}\left(\Omega_{\mathrm{m}}+\Omega_{\Lambda, 0}\right) \Omega_{\Lambda, 0}}+1=0$,

where $K \in[0,3)$.

The solution of the above equation is

$K_{\text {freeze }}=\frac{1}{3+\frac{1}{3 \Omega_{\gamma}\left(\Omega_{\mathrm{m}}+\Omega_{\Lambda, 0}\right) \Omega_{\Lambda, 0}}}$.

From Eq. (81), we can find an expression for a value of the scale factor for the freeze singularity

$a_{\text {freeze }}=\left(\frac{1-\Omega_{\Lambda, 0}}{8 \Omega_{\Lambda, 0}+\frac{1}{\Omega_{\gamma}\left(\Omega_{\mathrm{m}}+\Omega_{\Lambda, 0}\right)}}\right)^{\frac{1}{3}}$.

The relation between $a_{\text {freeze }}$ and positive $\Omega_{\gamma}$ is presented in Fig. 11.

The sudden singularity appears when $b=0$. This leaves us with the following algebraic equation:

$1+2 \Omega_{\gamma}\left(\Omega_{\mathrm{m}, 0} a^{-3}+\Omega_{\Lambda, 0}\right)(K+1)=0$.

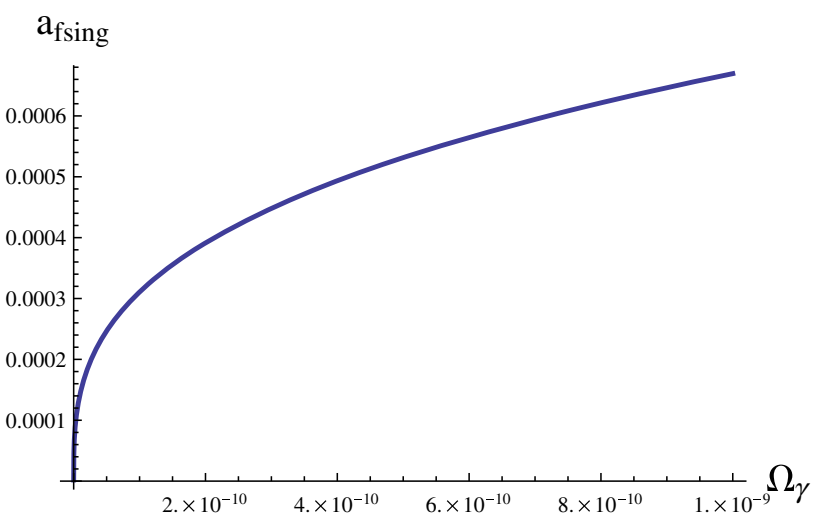

Fig. 11 Diagram of the relation between $a_{\text {sing }}$ and positive $\Omega_{\gamma}$. Note that in the limit $\Omega_{\gamma} \mapsto 0$ the singularity overlaps with a big-bang singularity

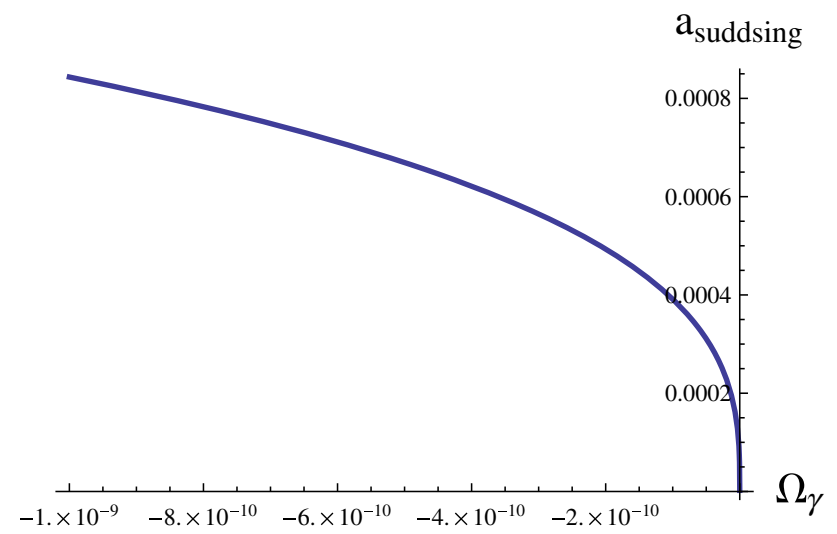

Fig. 12 Diagram of the relation between $a_{\text {sing }}$ and negative $\Omega_{\gamma}$. Note that in the limit $\Omega_{\gamma} \mapsto 0$ the singularity overlaps with a big-bang singularity

The above equation can be rewritten as

$1+2 \Omega_{\gamma}\left(\Omega_{\mathrm{m}, 0} a^{-3}+4 \Omega_{\Lambda, 0}\right)=0$.

From Eq. (84), we have the formula for the scale factor for a sudden singularity,

$a_{\text {sudden }}=\left(-\frac{2 \Omega_{\mathrm{m}, 0}}{\frac{1}{\Omega_{\gamma}}+8 \Omega_{\Lambda, 0}}\right)^{1 / 3}$,

which, in fact, becomes a (degenerate) critical point and a bounce at the same time. The relation between $a_{\text {sing }}$ and negative $\Omega_{\gamma}$ is presented in Fig. 12.

Let $V=-\frac{a^{2}}{2}\left(\Omega_{\gamma} \Omega_{\mathrm{ch}}^{2} \frac{(K-3)(K+1)}{2 b}+\Omega_{\mathrm{ch}}+\Omega_{k}\right)$. We can rewrite dynamical system (58)-(59) as

$a^{\prime}=x$,

$x^{\prime}=-\frac{\partial V(a)}{\partial a}$, 


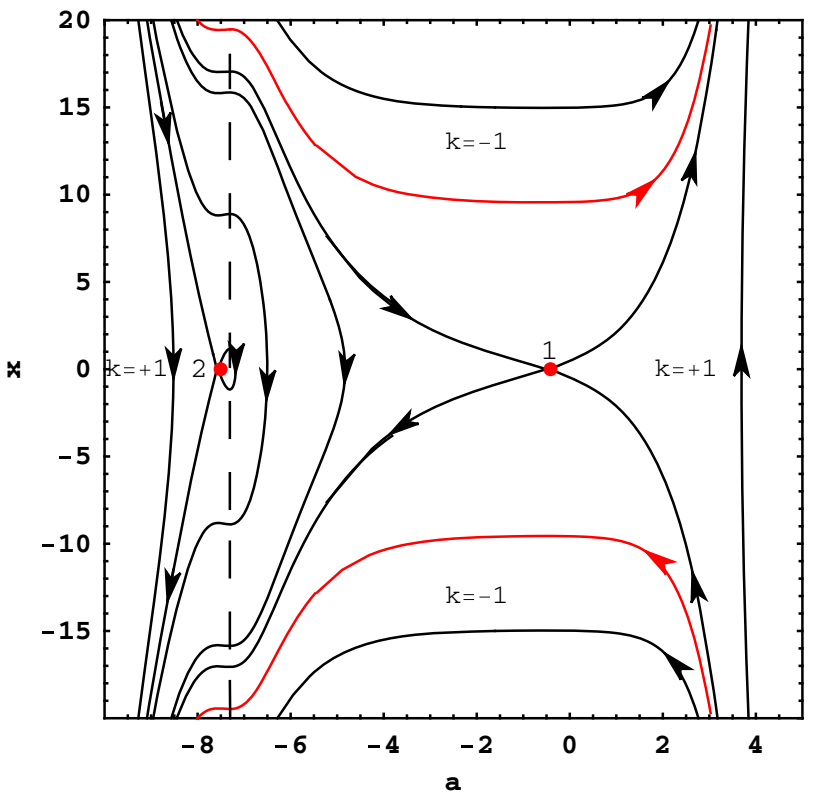

Fig. 13 The figure represents the phase portrait of the system (86-87) for positive $\Omega_{\gamma}$. The scale factor $a$ is in the logarithmic scale. The red trajectories represent the spatially flat universe. Trajectories under the top red trajectory and below the bottom red trajectory represent models with the negative spatial curvature. Trajectories between the top and bottom red trajectory represent models with the positive spatial curvature. The dashed line $2 b+d=0$ corresponds to the freeze singularity. The critical points (1) and (2) present two static Einstein universes. The phase portrait belongs to the class of sewn dynamical systems [49]

where $^{\prime} \equiv \frac{\mathrm{d}}{\mathrm{d} \sigma}=\frac{b+\frac{d}{2}}{b} \frac{\mathrm{d}}{\mathrm{d} \tau}$ is a new parametrization of time.

We can treat the dynamical system (86)-(87) as a sewn dynamical system $[47,48]$. In this case, we divide the phase portrait into two parts: the first part is for $a<a_{\text {sing }}$ and the second part is for $a>a_{\text {sing. }}$. Both parts are glued along the singularity.

For $a<a_{\text {sing, }}$, dynamical system (86)-(87) can be rewritten in the corresponding form,

$a^{\prime}=x$,

$x^{\prime}=-\frac{\partial V_{1}(a)}{\partial a}$,

where $V_{1}=V\left(-\eta\left(a-a_{s}\right)+1\right)$ and $\eta(a)$ notes the Heaviside function.

For $a>a_{\text {sing, }}$, in an analogous way, we get the following equations:

$a^{\prime}=x$,

$x^{\prime}=-\frac{\partial V_{2}(a)}{\partial a}$,

where $V_{2}=V \eta\left(a-a_{s}\right)$. The phase portraits, for dynamical system (86)-(87), are presented in Figs. 13 and 14. Figure

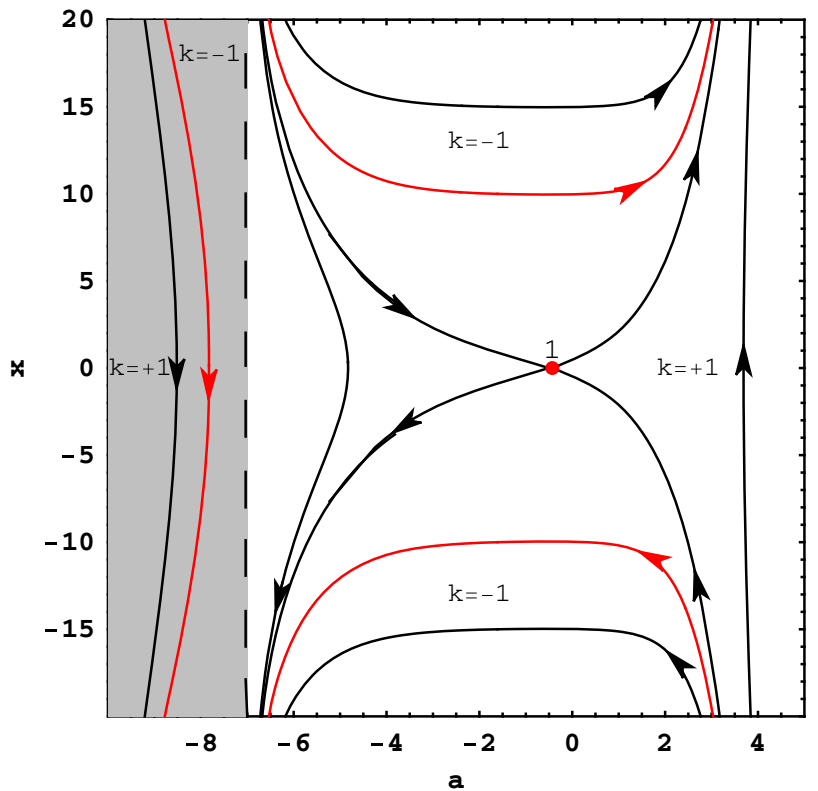

Fig. 14 The phase portrait of the system (86)-(87) for negative $\Omega_{\gamma}$. The scale factor $a$ is in logarithmic scale. The red trajectories represent a spatially flat universe. Trajectories under the top red trajectory and below the bottom red trajectory represent models with a negative spatial curvature. Trajectories between the top and bottom red trajectory represent models with the positive spatial curvature. The dashed line $b=0$ corresponds to the sudden singularity. The shaded region represents trajectories with $b<0$. If we assume that $f^{\prime}(R)>0$ then this region can be removed. The phase portrait possesses the symmetry $\dot{a} \rightarrow-\dot{a}$ and in consequence this singularity presents a bounce. This symmetry can be used to identify the corresponding points on the $b$-line. The critical point (1) represents the static Einstein universe. The phase portrait belongs to the class of sewn dynamical systems [49]

13 shows the phase portrait for positive $\Omega_{\gamma}$, while Fig. 14 shows the phase portrait for negative $\Omega_{\gamma}$.

In Fig. 13 there are two critical points labeled ' 1 ' and ' 2 ' at the finite domain. They are both saddle points. These critical points correspond to a maximum of the potential function. The saddle point ' 2 ' possesses the homoclinic closed orbit starting from it and returning to it. This orbit represents an emerging universe from the static Einstein universe and approaching it again. During the evolution this universe (orbit) goes two times through the freeze singularity. The region bounded by the homoclinic orbit contains closed orbits representing the oscillating universes. A diagram of the evolution of scale factor for closed orbit is presented by Fig. 15. It is also interesting that trajectories in the neighborhood of straight vertical line of freeze singularities undergo short time inflation $x=$ const. The characteristic number of e-foldings from $t_{\text {init }}$ to $t_{\text {fin }}$ of this inflation period $N=H_{\text {init }}\left(t_{\text {fin }}-t_{\text {init }}\right)$ (see Eq. (3.13) in [1]) with respect to $\Omega_{\gamma}$ is shown in Fig. 16. This figure illustrates the number of e-foldings is too small to obtain the inflation effect. 


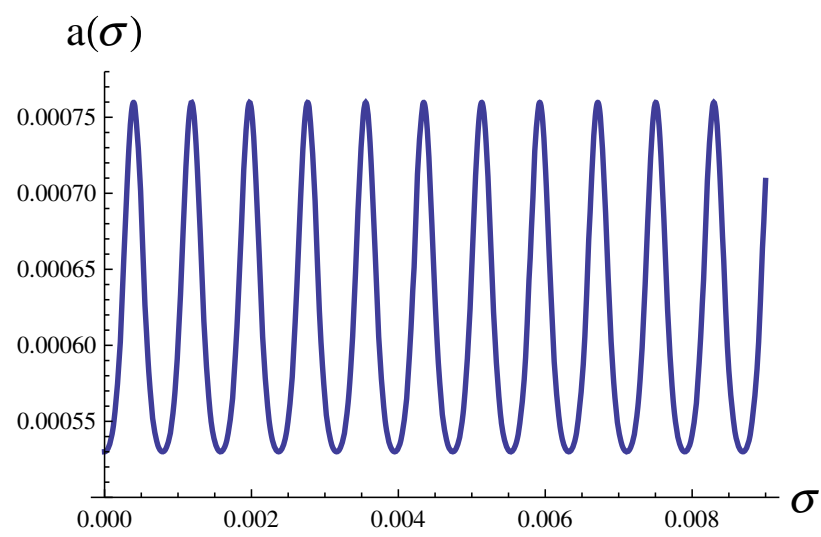

Fig. 15 Illustration of the evolution of $a(\sigma)$ for closed orbit which is contained by the homoclinic orbit, where $\sigma=\frac{b}{b+\frac{d}{2}} t$ is a reparametrization of time. We choose $\mathrm{s} \times \mathrm{Mpc} /(100 \times \mathrm{km})$ as a unit of $\sigma$

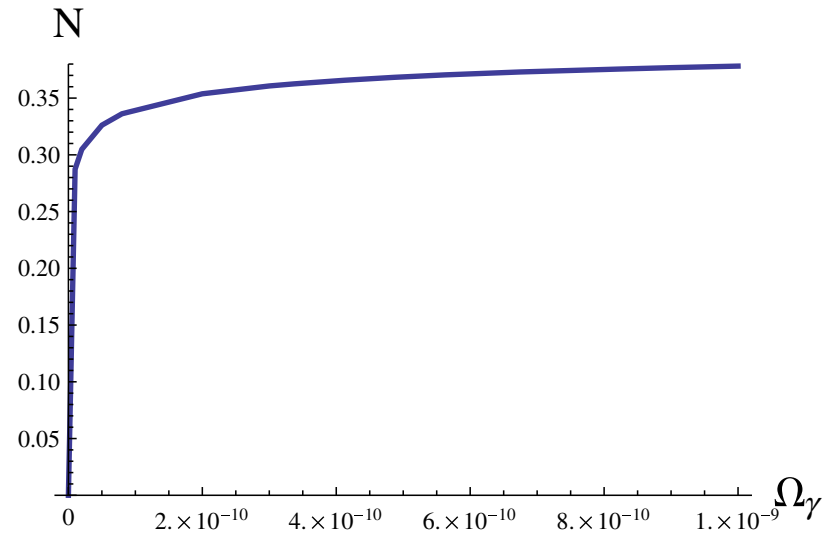

Fig. 16 Diagram of the relation between positive $\Omega_{\gamma}$ and the approximate number of e-foldings $N=H_{\text {init }}\left(t_{\text {fin }}-t_{\text {init }}\right)$ from $t_{\text {init }}$ to $t_{\text {fin }}$

\section{Observations}

In this paper we perform statistical analysis using the following astronomical observations: observations of 580 supernovae of type Ia, BAO, measurements of $H(z)$ for galaxies, Alcock-Paczyński test, measurements of CMB and lensing by Planck and low $\ell$ by WMAP.

The likelihood function for observations of supernovae of type Ia [50] is given by the following expression:

$\ln L_{\mathrm{SNIa}}=-\frac{1}{2}\left[A-B^{2} / C+\ln (C /(2 \pi))\right]$,

where $A=\left(\mu^{\text {obs }}-\mu^{\text {th }}\right) \mathbb{C}^{-1}\left(\mu^{\text {obs }}-\mu^{\text {th }}\right), B=\mathbb{C}^{-1}\left(\mu^{\text {obs }}-\right.$ $\left.\mu^{\text {th }}\right), C=\operatorname{Tr} \mathbb{C}^{-1}$ and $\mathbb{C}$ is a covariance matrix for observations of supernovae of type Ia. The distance modulus is defined by the formula $\mu^{\text {obs }}=m-M$ (where $m$ is the apparent magnitude and $M$ is the absolute magnitude of observations of supernovae of type Ia) and $\mu^{\text {th }}=5 \log _{10} D_{L}+25$ (where the luminosity distance is $\left.D_{L}=c(1+z) \int_{0}^{z} \frac{\mathrm{d} z^{\prime}}{H(z)}\right)$.
BAO observations such as Sloan Digital Sky Survey Release 7 (SDSS DR7) dataset at $z=0.275$ [51], 6dF Galaxy Redshift Survey measurements at redshift $z=0.1$ [52], and WiggleZ measurements at redshift $z=0.44,0.60,0.73$ [53] have the following likelihood function:

$\ln L_{\mathrm{BAO}}=-\frac{1}{2}\left(\mathbf{d}^{\mathrm{obs}}-\frac{r_{s}\left(z_{d}\right)}{D_{V}(\mathbf{z})}\right) \mathbb{C}^{-1}\left(\mathbf{d}^{\mathrm{obs}}-\frac{r_{s}\left(z_{d}\right)}{D_{V}(\mathbf{z})}\right)$,

where $r_{s}\left(z_{d}\right)$ is the sound horizon at the drag epoch [54,55].

For the Alcock-Paczynski test [56,57] we used the following expression for the likelihood function:

$\ln L_{A P}=-\frac{1}{2} \sum_{i} \frac{\left(A P^{\mathrm{th}}\left(z_{i}\right)-A P^{\mathrm{obs}}\left(z_{i}\right)\right)^{2}}{\sigma^{2}}$.

where $A P(z)^{\text {th }} \equiv \frac{H(z)}{z} \int_{0}^{z} \frac{\mathrm{d} z^{\prime}}{H\left(z^{\prime}\right)}$ and $A P\left(z_{i}\right)^{\text {obs }}$ are observational data [58-66].

The likelihood function for measurements of the Hubble parameter $H(z)$ of galaxies from [67-69] is given by the expression

$\ln L_{H(z)}=-\frac{1}{2} \sum_{i=1}^{N}\left(\frac{H\left(z_{i}\right)^{\mathrm{obs}}-H\left(z_{i}\right)^{\mathrm{th}}}{\sigma_{i}}\right)^{2}$.

In this paper, we use the likelihood function for observations of $\mathrm{CMB}$ [9] and lensing by Planck, and low- $\ell$ polarization from the WMAP (WP) in the following form:

$\ln L_{\mathrm{CMB}+\text { lensing }}=-\frac{1}{2}\left(\mathbf{x}^{\mathrm{th}}-\mathbf{x}^{\mathrm{obs}}\right) \mathbb{C}^{-1}\left(\mathbf{x}^{\mathrm{th}}-\mathbf{x}^{\mathrm{obs}}\right)$,

where $\mathbb{C}$ is the covariance matrix with the errors, $\mathbf{x}$ is a vector of the acoustic scale $l_{A}$, the shift parameter $R$ and $\Omega_{b} h^{2}$ where

$l_{A}=\frac{\pi}{r_{S}\left(z^{*}\right)} c \int_{0}^{z^{*}} \frac{\mathrm{d} z^{\prime}}{H\left(z^{\prime}\right)}$

$R=\sqrt{\Omega_{\mathrm{m}, 0} H_{0}^{2}} \int_{0}^{z^{*}} \frac{\mathrm{d} z^{\prime}}{H\left(z^{\prime}\right)}$,

where $z^{*}$ is the redshift of the epoch of the recombination [54].

The total likelihood function is expressed in the following form:

$L_{\text {tot }}=L_{\mathrm{SNIa}} L_{\mathrm{BAO}} L_{\mathrm{AP}} L_{H(z)} L_{\mathrm{CMB}+\text { lensing }}$.

To estimate model parameters, we use our own code CosmoDarkBox. The Metropolis-Hastings algorithm [70,71] is used in this code. 
Table 1 The best fit and errors for the estimated model for the positive $\Omega_{\gamma}$ with $\Omega_{\mathrm{m}, 0}$ from the interval $(0.27,0.33), \Omega_{\gamma}$ from the interval $\left(0.0,2.6 \times 10^{-9}\right)$ and $H_{0}$ from the interval [66.0 $(\mathrm{km} /(\mathrm{s} \mathrm{Mpc})), 70.0$ $(\mathrm{km} /(\mathrm{s} \mathrm{Mpc}))] . \Omega_{\mathrm{b}, 0}$ is assumed as 0.048468 . The redshift of matter- radiation equality is assumed as $3395 . H_{0}$, in the table, is expressed in $\mathrm{km} /(\mathrm{s} \mathrm{Mpc})$. The value of reduced $\chi^{2}$ of the best fit of our model is equal 0.187066 (for the $\Lambda$ CDM model 0.186814 )

\begin{tabular}{llll}
\hline Parameter & Best fit & $68 \%$ CL & $95 \%$ CL \\
\hline$H_{0}$ & 68.10 & +1.07 & +1.55 \\
& & -1.24 & 1.82 \\
$\Omega_{\mathrm{m}, 0}$ & 0.3011 & +0.0145 & +0.0217 \\
$\Omega_{\gamma}$ & & -0.0138 & -0.0201 \\
& $9.70 \times 10^{-11}$ & $+1.3480 \times 10^{-9}$ & $+2.2143 \times 10^{-9}$ \\
& & $-9.70 \times 10^{-11}$ & $-9.70 \times 10^{-11}$ \\
\hline
\end{tabular}

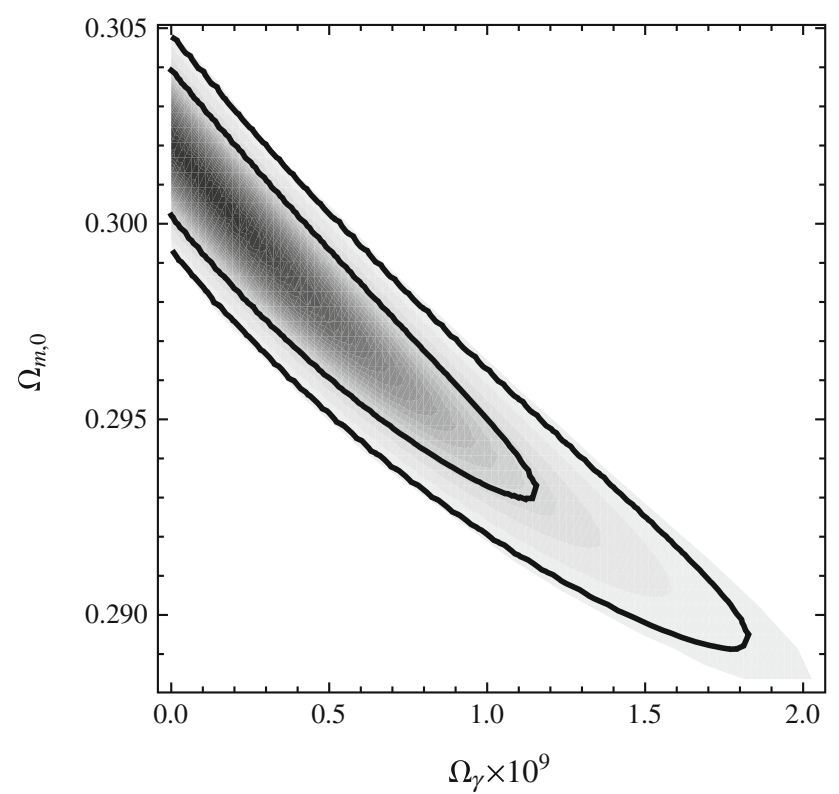

Fig. 17 The intersection of the likelihood functions of two model parameters $\left(\Omega_{\gamma}, \Omega_{m, 0}\right)$ with the marked 68 and $95 \%$ confidence levels

Table 1 shows the values of parameters for the best fit with errors. Figures 17 and 18 show the intersection of a likelihood function with the 68 and $95 \%$ confidence level projections on the $\left(\Omega_{\gamma}, \Omega_{\mathrm{m}, 0}\right)$ and $\left(\Omega_{\gamma}, H_{0}\right)$ planes.

In this paper, we use the Bayesian information criterion (BIC) [72,73], for comparison of our model with the $\Lambda \mathrm{CDM}$ model. The expression for BIC is defined as

$\mathrm{BIC}=\chi^{2}+j \ln n$,

where $\chi^{2}$ is the value of $\chi^{2}$ in the best fit, $j$ is the number of model parameters (our model has three parameters, $\Lambda \mathrm{CDM}$ model has two parameters) and $n$ is the number of data points $(n=625)$ which are used in the estimation.

For our model, the value of BIC is equal 135.668 and for the $\Lambda \mathrm{CDM}$ model $\mathrm{BIC}_{\Lambda \mathrm{CDM}}=129.261$. So $\triangle \mathrm{BIC}=\mathrm{BIC}-$ $\mathrm{BIC}_{\Lambda \mathrm{CDM}}$ is equal 6.407. The evidence for the model is

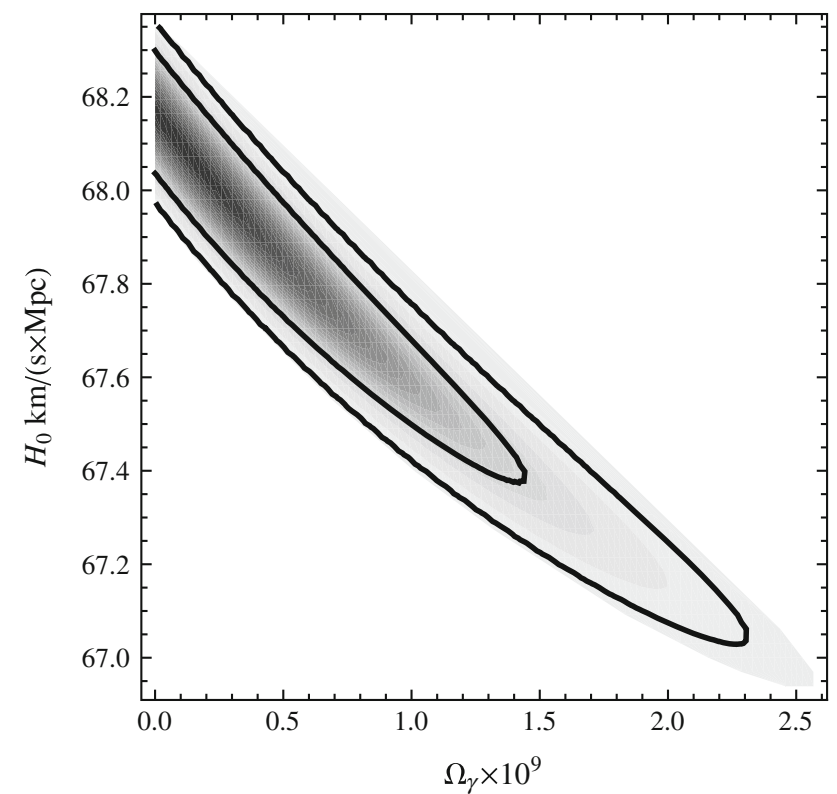

Fig. 18 The intersection of the likelihood functions of two model parameters $\left(\Omega_{\gamma}, H_{0}\right)$ with the marked 68 and $95 \%$ confidence levels

strong [73] if $\triangle \mathrm{BIC}$ is higher than 6 . So, in comparison to our model, the evidence in favor of the $\Lambda \mathrm{CDM}$ model is strong, but we cannot absolutely reject our model.

\section{Conclusions}

In this paper, we demonstrated that evolution of the Starobinsky model with a quadratic term $R^{2}$ gives rise to the description of dynamics in terms of piecewise-smooth dynamical systems, i.e., systems whose the phase space is partitioned into different regions, each of them associated to a different smooth functional form of the system of a Newtonian type. Different regions of the phase space correspond to different forms of the potential separated by singularities of the type of poles.

Our idea was to obtain inflation as an endogenous effect of the dynamics in the Palatini formalism. While the effect of 
inflation appears in the model under consideration a sufficient number of e-folds are not achieved and the additional effect of amplification is required. Note that this type of inflation is a realization of the idea of singular inflation [74-77]. In our model inflation is driven by the freeze degenerate singularity (the extension of a type III isolated singularity).

We show that the dynamics of the model can be analyzed in terms of two-dimensional dynamical systems of the Newtonian type. In this approach, in the diagram of the potential of a fictitious particle, the evolution of the universe contains all information which is needed for an investigation of singularities in the model. Note that they are not isolated singularities which were classified into five types but rather double singularities glued in one point of the evolution at

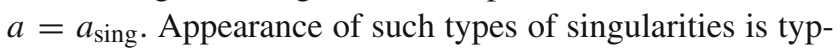
ical for piecewise-smooth dynamics describing the model evolution. We call this type sewn singularities in analogy to sewn dynamical systems [78,79].

We investigated the model with $f(\hat{R})=\hat{R}+\gamma \hat{R}^{2}$, where $\gamma$ assumes the positive or negative values. While the dynamics of this class of models depend crucially on the sign of the parameter $\gamma$ in the early universe for the late time we obtain the behavior consistent with the $\Lambda \mathrm{CDM}$ model.

Note that in the model with positive $\gamma$, the phase space is a sum of two disjoint domains which boundary represents the double freeze singularity (cf. Fig. 13). In the first domain the evolution starts from a big bang followed by the deceleration phase; then it changes to acceleration (early acceleration $\equiv$ inflation) after reaching a maximum of the potential function. In the second domain, on the right from the vertical line of the freeze singularity, the universe decelerates and after reaching another maximum starts to accelerate again. This last eternal acceleration corresponds to the present day epoch called the dark energy domination epoch. Two phases of deceleration and two phases of acceleration are key ingredients of our model. While the first phase models a transition from the matter domination epoch to inflation the second phase models a transition from the second matter dominated epoch toward the present day acceleration.

As De Felice and Tsujikawa have noted [1, p. 24] the applications of $f(R)$ theories should be focused on construct of viable cosmological models, for which a sequence of radiation, matter and accelerating epochs is realized. All these epochs are also presented in the model under consideration but, for negative $\gamma$ (negative squared $M^{2}$ for the scalar field), some difficulties appear in the interpretation of the phase space domain $\left\{a: a<a_{\text {sing }}\right\}$. The size of this domain will depend on the value of the parameter $\Omega_{\gamma}$ and this domain vanishes as we are going toward $\Omega_{\gamma}$ equal zero.

On the other hand it is well known that violation of condition $f_{\hat{R} \hat{R}}^{\prime \prime}>0$ gives rise to the negative values of $M^{2}$. We do not assume this condition but we require that $f_{\hat{R}}^{\prime}>0$ to avoid the appearance of ghosts (see Sect. 7.4 in [1]). In our case, statistical analysis favors a model with $f_{\hat{R}}^{\prime}>0\left(\Omega_{\gamma}>0\right)$ rather than a model with $f_{\hat{R}}^{\prime}<0\left(\Omega_{\gamma}<0\right)$. In other words, statistical analysis favors the case without ghosts.

In order to obtain deeper insight into the model we have also performed complementary investigations in the Einstein frame. In this case we find that the model is reduced to the FRW cosmological model with the selfinteracting scalar field and the vanishing part of the kinetic energy. Therefore from the Palatini formulation we obtain directly the form of the potential and the (implicit) functional dependence between the scalar field and the scale factor. Moreover, we obtain the parametrization of the decaying cosmological constant.

Due to a time-dependent cosmological constant the model evolution can be described in terms of an interaction between the matter and the decaying lambda terms. We study how the energy is transferred between the sectors and how the standard scaling relation for matter is modified.

We pointed out that the consideration of the Starobinsky model in the Einstein frame gives rise to new interesting properties from the cosmological point of view; similar to the original (metric) the Starobinsky model is very important for the explanation of inflation. The model under the consideration gives rise analogously to the running cosmological term. This fact seems to be interesting in the context of an explanation of the cosmological constant problem.

Detailed conclusions coming from our analysis are the following:

- We show that the interaction between two sectors: the matter and the decaying vacuum, appears naturally in the Einstein frame. For the model formulated in the Jordan frame this interaction is absent.

- Inflation appears in our model formulated in the Einstein frame, when the parameter $\gamma$ is close to zero and the density of matter is negligible in comparison to $\bar{\rho}_{\Phi}$.

- In our model in the Einstein frame, the potential $\bar{U}(\Phi)$ has the same shape as the Starobinsky potential and has the minimum for $\Phi=1+8 \gamma \lambda$.

- While the freeze double singularities appear in our model in the Jordan frame there are no such singularities in the dynamics of the model in the Einstein frame.

- If $\Omega_{\gamma}$ is small, then $a_{\text {sing }}=\left(-\frac{2 \Omega_{\mathrm{m}, 0}}{\frac{1}{\Omega_{\gamma}}+8 \Omega_{\Lambda, 0}}\right)^{1 / 3}$ for negative $\Omega_{\gamma}$ and $a_{\text {sing }}=\left(\frac{1-\Omega_{\Lambda, 0}}{8 \Omega_{\Lambda, 0}+\frac{1}{\Omega_{\gamma}\left(\Omega_{m}+\Omega_{\Lambda, 0}\right)}}\right)^{\frac{1}{3}}$ for positive $\Omega_{\gamma}$. These values define the natural scale at which singularities appear in the model under consideration with the negative or positive value of $\gamma$ parameter. It seems to be natural to identify this scale with a cut off at which the model can be treated as some kind of effective theory.

- In both the cases of a negative and positive $\gamma$ one deals with a finite scale factor singularity. For negative $\gamma$ it 
is a double sudden singularity which meets the future singularity of a contracting model before the bounce with the initial singularity in the expanding model. The sewn evolutionary scenarios reveal the presence of a bounce during the cosmic evolution.

- In the context of the Starobinsky model in the Palatini formalism we found a new type of double singularity beyond the well-known classification of isolated singularities.

- The phase portrait for the model with a positive value of $\gamma$ is equivalent to the phase portrait of the $\Lambda \mathrm{CDM}$ model (following dynamical system theory [80] equivalence assumes the form of topological equivalence established by a homeomorphism). There is only a quantitative difference related with the presence of the non-isolated freeze singularity. The scale of the appearance of this type singularity can also be estimated and be cast in terms of the redshift $z_{\text {freeze }}=\Omega_{\gamma}^{-1 / 3}$.

- We estimated the model parameters using astronomical data and conclude that positive $\Omega_{\gamma}$ is favored by the best fit value; still the model without $\hat{R}^{2}$ term is statistically admitted.

In our model, the best fit value of $\Omega_{\gamma}$ is equal $9.70 \times$ $10^{-11}$ and positive $\Omega_{\gamma}$ parameter belongs to the interval $\left(0,2.2143 \times 10^{-9}\right)$ at $2-\sigma$ level. This mean that the positive value of $\Omega_{\gamma}$ is more favored by astronomical data than the negative value of $\Omega_{\gamma}$. The difference between values of BIC for our model and the $\Lambda$ CDM model is equal 6.407. So, in comparison to our model, the evidence in favor of the $\Lambda \mathrm{CDM}$ model is strong. But one cannot absolutely reject the model.

Note added in proof After completing the paper we found a paper by Faraoni and Cardini where freeze singularities have been analyzed in a different context, both from point particle and cosmological perspectives [81].

Acknowledgements $\mathrm{AB}$ and $\mathrm{MS}$ would like to thank Salvatore Capozziello for encouraging us to consider this model in different frames. The work has been supported by the Polish National Science Centre (NCN), project DEC-2013/09/B/ ST2/03455.

Open Access This article is distributed under the terms of the Creative Commons Attribution 4.0 International License (http://creativecomm ons.org/licenses/by/4.0/), which permits unrestricted use, distribution, and reproduction in any medium, provided you give appropriate credit to the original author(s) and the source, provide a link to the Creative Commons license, and indicate if changes were made.

Funded by SCOAP ${ }^{3}$.

\section{References}

1. A. De Felice, S. Tsujikawa, f(R) Theories. Living Rev. Rel. 13, 3 (2010). arXiv: 1002.4928

2. A.A. Starobinsky, A new type of isotropic cosmological models without singularity. Phys. Lett. B 91, 99-102 (1980)
3. A.H. Guth, The inflationary universe: a possible solution to the horizon and flatness problems. Phys. Rev. D 23, 347-356 (1981)

4. A.D. Linde, Eternally existing selfreproducing chaotic inflationary universe. Phys. Lett. B 175, 395-400 (1986)

5. S. Nojiri, S.D. Odintsov, Introduction to modified gravity and gravitational alternative for dark energy. Int. J. Geom. Method Mod. Phys. 4, 115 (2007). arXiv: hep-th/0601213

6. T.P. Sotiriou, V. Faraoni, f(R) Theories of gravity. Rev. Mod. Phys. 82, 451-497 (2010). arXiv:0805.1726

7. V.F. Mukhanov, G.V. Chibisov, Quantum fluctuations and a nonsingular universe. JETP Lett. 33, 532-535 (1981). Pisma Zh. Eksp. Teor. Fiz.33,549(1981)

8. A.A. Starobinsky, The perturbation spectrum evolving from a nonsingular initially de-Sitter cosmology and the microwave background anisotropy. Sov. Astron. Lett. 9, 302 (1983)

9. Planck Collaboration, P.A.R. Ade, Planck 2015 results. XIV. Dark energy and modified gravity. Astron. Astrophys. 594, A14 (2016). arXiv: 1502.01590

10. C. Cheng, Q.-G. Huang, Y.-Z. Ma, Constraints on single-field inflation with WMAP. SPT and ACT data-a last-minute stand before Planck. JCAP 1307, 018 (2013). arXiv: 1303.4497

11. Q.-G. Huang, A polynomial $f(R)$ inflation model. JCAP 1402, 035 (2014). arXiv:1309.3514

12. L.A. Kofman, A.D. Linde, A.A. Starobinsky, Inflationary universe generated by the combined action of a scalar field and gravitational vacuum polarization. Phys. Lett. B 157, 361-367 (1985)

13. S.V. Ketov, A.A. Starobinsky, Embedding $\left(R+R^{2}\right)$-inflation into supergravity. Phys. Rev. D 83, 063512 (2011). arXiv: 1011.0240

14. S.A. Appleby, R.A. Battye, A.A. Starobinsky, Curing singularities in cosmological evolution of F(R) gravity. JCAP 1006, 005 (2010). arXiv:0909.1737

15. S. Capozziello, M. De Laurentis, S. Nojiri, S.D. Odintsov, Classifying and avoiding singularities in the alternative gravity dark energy models. Phys. Rev. D 79, 124007 (2009). arXiv:0903.2753

16. A. Alho, S. Carloni, C. Uggla, On dynamical systems approaches and methods in $f(R)$ cosmology. JCAP 1608(08), 064 (2016). arXiv:1607.05715

17. S. Capozziello, M.F. De Laurentis, L. Fatibene, M. Ferraris, S. Garruto, Extended cosmologies. SIGMA 12, 006 (2016). arXiv: 1509.08008

18. S. Capozziello, P. Martin-Moruno, C. Rubano, Physical nonequivalence of the Jordan and Einstein frames. Phys. Lett. B 689, 117-121 (2010). arXiv:1003.5394

19. S.M. Carroll, A. De Felice, V. Duvvuri, D.A. Easson, M. Trodden, M.S. Turner, The cosmology of generalized modified gravity models. Phys. Rev. D 71, 063513 (2005). arXiv: astro-ph/0410031

20. A. Borowiec, M. Kamionka, A. Kurek, M. Szydlowski, Cosmic acceleration from modified gravity with Palatini formalism. JCAP 1202, 027 (2012). arXiv: 1109.3420

21. G.J. Olmo, Palatini approach to modified gravity: $f(R)$ theories and beyond. Int. J. Mod. Phys. D 20, 413-462 (2011). arXiv:1101.3864

22. G.J. Olmo, Post-Newtonian constraints on $f(R)$ cosmologies in metric and Palatini formalism. Phys. Rev. D 72, 083505 (2005). arXiv: gr-qc/0505135

23. G.J. Olmo, The gravity Lagrangian according to solar system experiments. Phys. Rev. Lett. 95, 261102 (2005). arXiv: gr-qc/0505101

24. C. Barragan, G.J. Olmo, Isotropic and anisotropic bouncing cosmologies in Palatini gravity. Phys. Rev. D 82, 084015 (2010). arXiv: 1005.4136

25. C. Barragan, G.J. Olmo, H. Sanchis-Alepuz, Bouncing cosmologies in Palatini $\mathrm{f}(\mathrm{R})$ gravity. Phys. Rev. D 80, 024016 (2009). arXiv:0907.0318

26. C. Bejarano, G.J. Olmo, D. Rubiera-Garcia, What is a singular black hole beyond general relativity? Phys. Rev. D 95, 064043 (2017). arXiv:1702.01292 
27. C. Bambi, A. Cardenas-Avendano, G.J. Olmo, D. Rubiera-Garcia, Wormholes and nonsingular spacetimes in Palatini $f(R)$ gravity. Phys. Rev. D 93, 064016 (2016). arXiv:1511.03755

28. G.J. Olmo, D. Rubiera-Garcia, Nonsingular black holes in $f(R)$ theories. Universe 1, 173-185 (2015). arXiv: 1509.02430

29. G.J. Olmo, D. Rubiera-Garcia, Nonsingular black holes in quadratic Palatini gravity. Eur. Phys. J. C 72, 2098 (2012). arXiv: 1112.0475

30. G.J. Olmo, D. Rubiera-Garcia, Palatini $f(R)$ black holes in nonlinear electrodynamics. Phys. Rev. D 84, 124059 (2011). arXiv: 1110.0850

31. E.E. Flanagan, Palatini form of 1/R gravity. Phys. Rev. Lett. 92, 071101 (2004). arXiv: astro-ph/0308111

32. E.E. Flanagan, The conformal frame freedom in theories of gravitation. Class. Quantum Gravity 21, 3817 (2004). arXiv: gr-qc/0403063

33. F.A. Teppa Pannia, F. Garcia, S.E. Perez Berliaffa, M. Orellana, G.E. Romero, Structure of compact stars in R-squared Palatini gravity. Gen. Relativ. Gravity 49, 25 (2017). arXiv: 1607.03508

34. T. Koivisto, Covariant conservation of energy momentum in modified gravities. Class. Quantum Gravity 23, 4289-4296 (2006). arXiv: gr-qc/0505128

35. L. Fernandez-Jambrina, R. Lazkoz, Classification of cosmological milestones. Phys. Rev. D 74, 064030 (2006). arXiv: gr-qc/0607073

36. S. Bahamonde, S.D. Odintsov, V.K. Oikonomou, P.V. Tretyakov, Deceleration versus acceleration universe in different frames of $F(R)$ gravity. Phys. Lett. B 766, 225-230 (2017). arXiv: 1701.02381

37. M.P. Dabrowski, J. Garecki, D.B. Blaschke, Conformal transformations and conformal invariance in gravitation. Ann. Phys. 18, 13-32 (2009). arXiv:0806.2683

38. BICEP2 Collaboration, Ade, P.A.R., et al.: Detection of $B$-mode polarization at Degree Angular Scales by BICEP2. Phys. Rev. Lett.112(24), 241101 (2014). arXiv: 1403.3985

39. O. Hrycyna, M. Szydlowski, M. Kamionka, Dynamics and cosmological constraints on Brans-Dicke cosmology. Phys. Rev. D 90(12), 124040 (2014). arXiv:1404.7112

40. S. Nojiri, S.D. Odintsov, S. Tsujikawa, Properties of singularities in (phantom) dark energy universe. Phys. Rev. D 71, 063004 (2005). arXiv: hep-th/0501025

41. J.D. Barrow, Sudden future singularities. Class. Quantum Gravity 21, L79-L82 (2004). arXiv: gr-qc/0403084

42. M. Bouhmadi-Lopez, P.F. Gonzalez-Diaz, P. Martin-Moruno, Worse than a big rip? Phys. Lett. B 659, 1-5 (2008). arXiv:gr-qc/0612135

43. A. Krolak, Towards the proof of the cosmic censorship hypothesis. Class. Quantum Gravity 3, 267-280 (1986)

44. A. Borowiec, A. Stachowski, M. Szydlowski, Inflationary cosmology with Chaplygin gas in Palatini formalism. JCAP 1601(01), 040 (2016). arXiv: 1512.01199

45. Sbierski, J.: The $C^{0}$-inextendibility of the Schwarzschild spacetime and the spacelike diameter in Lorentzian geometry (2015). arXiv:1507.00601

46. Galloway, G., Ling, E.: Some remarks on the $C^{0}$-(in)extendibility of spacetimes (2016). arXiv: 1610.03008

47. O. Hrycyna, M. Szydlowski, Non-minimally coupled scalar field cosmology on the phase plane. JCAP 0904, 026 (2009). arXiv:0812.5096

48. G .F .R. Ellis, E. Platts, D. Sloan, Current observations with a decaying cosmological constant allow for chaotic cyclic cosmology. JCAP 1604(04), 026 (2016). arXiv: 1511.03076

49. Bautin, N.N., Leontovich, I.A. (eds.): Methods and Techniques for Qualitative Analysis of Dynamical Systems on the Plane. Nauka, Moscow (1976). (In Russian)

50. N. Suzuki et al., The Hubble space telescope cluster supernova survey: V. Improving the dark energy constraints above $z>1$ and building an early-type-hosted supernova sample. Astrophys. J. 746, 85 (2012). arXiv: 1105.3470

51. S.D.S.S. Collaboration, W.J. Percival et al., Baryon acoustic oscillations in the sloan digital sky survey data release 7 galaxy sample. Mon. Not. R. Astron. Soc. 401, 2148-2168 (2010). arXiv:0907.1660

52. F. Beutler, C. Blake, M. Colless, D.H. Jones, L. Staveley-Smith, L. Campbell, Q. Parker, W. Saunders, F. Watson, The 6dF galaxy survey: baryon acoustic oscillations and the local Hubble constant. Mon. Not. R. Astron. Soc. 416, 3017-3032 (2011). arXiv: 1106.3366

53. C. Blake et al., The WiggleZ dark energy survey: Joint measurements of the expansion and growth history at $z<1$. Mon. Not. R. Astron. Soc. 425, 405-414 (2012). arXiv:1204.3674

54. W. Hu, N. Sugiyama, Small scale cosmological perturbations: an analytic approach. Astrophys. J. 471, 542-570 (1996). arXiv: astro-ph/9510117

55. D.J. Eisenstein, W. Hu, Baryonic features in the matter transfer function. Astrophys. J. 496, 605 (1998). arXiv: astro-ph/9709112

56. C. Alcock, B. Paczynski, An evolution free test for non-zero cosmological constant. Nature 281, 358-359 (1979)

57. M. Lopez-Corredoira, Alcock-Paczynski cosmological test. Astrophys. J. 781(2), 96 (2014). arXiv:1312.0003

58. P.M. Sutter, G. Lavaux, B.D. Wandelt, D.H. Weinberg, A first application of the Alcock-Paczynski test to stacked cosmic voids. Astrophys. J. 761, 187 (2012). arXiv: 1208.1058

59. C. Blake et al., The WiggleZ dark energy survey: measuring the cosmic expansion history using the Alcock-Paczynski test and distant supernovae. Mon. Not. R. Astron. Soc. 418, 1725-1735 (2011). arXiv: 1108.2637

60. N.P. Ross et al., The 2dF-SDSS LRG and QSO survey: the 2-point correlation function and redshift-space distortions. Mon. Not. R. Astron. Soc. 381, 573-588 (2007). arXiv: astro-ph/0612400

61. C. Marinoni, A. Buzzi, A geometric measure of dark energy with pairs of galaxies. Nature 468(7323), 539-541 (2010)

62. J. da Angela, P.J. Outram, T. Shanks, Constraining beta(z) and Omega $\mathrm{O}(\mathrm{m})$ from redshift-space distortions in $\mathrm{z} 3$ galaxy surveys. Mon. Not. R. Astron. Soc. 361, 879-886 (2005). arXiv: astro-ph/0505469

63. P.J. Outram, T. Shanks, B.J. Boyle, S.M. Croom, F. Hoyle, N.S. Loaring, L. Miller, R.J. Smith, The 2 df qso redshift survey. 13. A measurement of lambda from the qso power spectrum. Mon. Not. R. Astron. Soc. 348, 745 (2004). arXiv: astro-ph/0310873

64. L. Anderson et al., The clustering of galaxies in the SDSS-III baryon oscillation spectroscopic survey: baryon acoustic oscillations in the data release 9 spectroscopic galaxy sample. Mon. Not. R. Astron. Soc. 427(4), 3435-3467 (2013). arXiv:1203.6594

65. I. Paris et al., The Sloan Digital Sky Survey quasar catalog: ninth data release. Astron. Astrophys. 548, A66 (2012). arXiv:1210.5166

66. S.D.S.S. Collaboration, D.P. Schneider et al., The Sloan Digital Sky Survey quasar catalog V. Seventh data release. Astron. J. 139, 2360-2373 (2010). arXiv:1004.1167

67. J. Simon, L. Verde, R. Jimenez, Constraints on the redshift dependence of the dark energy potential. Phys. Rev. D 71, 123001 (2005). arXiv: astro-ph/0412269

68. D. Stern, R. Jimenez, L. Verde, M. Kamionkowski, S.A. Stanford, Cosmic chronometers: constraining the equation of state of dark energy. I: H(z) measurements. JCAP 1002, 008 (2010). arXiv:0907.3149

69. M. Moresco et al., Improved constraints on the expansion rate of the Universe up to $\mathrm{z} 1.1$ from the spectroscopic evolution of cosmic chronometers. JCAP 1208, 006 (2012). arXiv:1201.3609

70. N. Metropolis, A.W. Rosenbluth, M.N. Rosenbluth, A.H. Teller, E. Teller, Equation of state calculations by fast computing machines. J. Chem. Phys. 21, 1087-1092 (1953) 
71. W.K. Hastings, Monte carlo sampling methods using markov chains and their applications. Biometrika 57, 97-109 (1970)

72. G. Schwarz, Estimating the dimension of a model. Ann. Stat. 6(2), 461-464 (1978)

73. R.E. Kass, A.E. Raftery, Bayes factors. J. Am. Stat. Assoc. 90, 773-795 (1995)

74. J.D. Barrow, A.A.H. Graham, Singular inflation. Phys. Rev. D 91(8), 083513 (2015). arXiv:1501.04090

75. Bamba, K.: Inflationary universe in fluid description (2016). arXiv: 1601.04773

76. S. Nojiri, S.D. Odintsov, V.K. Oikonomou, Singular inflation from generalized equation of state fluids. Phys. Lett. B 747, 310-320 (2015). arXiv: 1506.03307
77. S.D. Odintsov, V.K. Oikonomou, Singular inflationary universe from $F(R)$ gravity. Phys. Rev. D 92(12), 124024 (2015). arXiv: 1510.04333

78. Z.T. Zhusubaliyev, E. Mosekilde, Bifurcations and Chaos in Piecewise-Smooth Dynamical Systems (World Scientific, Singapore, 2003)

79. R. Leine, H. Nijmeijer, Dynamics and Bifurcations of Non-Smooth Mechanical Systems (Springer, Berlin, 2004)

80. L. Perko et al., Differential Equations and Dynamical Systems, vol. 7, 3rd edn., Texts in Applied Mathematics (Springer, New York, 2001)

81. Faraoni, V., Cardini, A.M.: Analogues of glacial valley profiles in particle mechanics and in cosmology (2016). arXiv: 1608.02542 